\title{
Do the laws of physics forbid the operation of time machines?
}

\author{
John Earman • Christopher Smeenk • \\ Christian Wüthrich
}

Received: 28 July 2006 / Accepted: 1 April 2008 / Published online: 7 May 2008

(C) Springer Science+Business Media B.V. 2008

\begin{abstract}
We address the question of whether it is possible to operate a time machine by manipulating matter and energy so as to manufacture closed timelike curves. This question has received a great deal of attention in the physics literature, with attempts to prove no-go theorems based on classical general relativity and various hybrid theories serving as steps along the way towards quantum gravity. Despite the effort put into these no-go theorems, there is no widely accepted definition of a time machine. We explain the conundrum that must be faced in providing a satisfactory definition and propose a resolution. Roughly, we require that all extensions of the time machine region contain closed timelike curves; the actions of the time machine operator are then sufficiently "potent" to guarantee that closed timelike curves appear. We then review no-go theorems based on classical general relativity, semi-classical quantum gravity, quantum field theory on curved spacetime, and Euclidean quantum gravity. Our verdict on the question of our title is that no result of sufficient generality to underwrite a confident "yes" has been proven. Our review of the no-go results does, however, highlight several foundational problems at the intersection of general relativity and quantum physics that lend substance to the search for an answer.
\end{abstract}

\footnotetext{
J. Earman

Department of History and Philosophy of Science, University of Pittsburgh, 1017 Cathedral of Learning, Pittsburgh, PA 15260, USA

C. Smeenk $(\varangle)$

Department of Philosophy, The University of Western Ontario, Talbot College, London, Ontario, Canada N6A 3K7

e-mail: csmeenk2@uwo.ca

C. Wüthrich

Department of Philosophy, University of California, 9500 Gilman Dr. La Jolla, San Diego, CA 92093-0119, USA
} 
Keywords General relativity - Time travel - Time machines - QFT on curved spacetime $\cdot$ Causality $\cdot$ Closed timelike curves

\section{Introduction}

The twin topics of time travel and time machines continue to exert a hold on the popular imagination. (Recent popularizations include the books by Davies (2002), Gott (2001), and Randles (2005). The science section of the New York Times occasionally runs articles on time travel and time machines; see Overbye (2005).) The philosophical literature records a steady trickle and occasional spikes of articles on the these topics. (Volume 88, number 3 of the Monist (2005) is devoted to the topic of time travel.) And Visser (2003) estimates that as of that date the physics literature contained over two hundred articles on time travel and time machines, most of them of recent vintage. ${ }^{1}$ The popular literature can hardly be faulted for its simplifications and enthusiasms since its purpose is fire the imagination rather than dull it with details and qualms. The philosophical literature, however, is another story. It has focused in part on analyzing the "grandfather paradox" and related issues. We find fault with this literature in that, first, it often fails to identify distinctive problems related to time travel, rather than re-packaging the conflict between free will and determinism. Second, it is unclear what a time traveller's inability to kill Grandpa implies regarding the physical possibility or plausibility of time travel. There are certainly subtle and interesting issues regarding modality here, but (as we hope to illustrate below) philosophers have more to gain from a study of how time travel has been handled in the physics literature than from continued analysis of the grandfather paradox. ${ }^{2}$ In addition, philosophers have largely neglected the distinctive issues raised by the physics literature on time machines. These issues are independent of the time travel paradoxes since they are concerned with attempts to establish-or evade-"no-go" results for time machines that focus on what happens before closed timelike curves (CTCs) are formed. This is not to say that the physics literature is unproblematic. For one thing, a reader will search this literature in vain for a satisfactory definition of "time machine" in terms of spacetime structure. ${ }^{3}$ For reasons to be explained below, this is less scandalous than it sounds. But there is obviously a task here that calls for the kind of analysis that is stock-in-trade for philosophy.

The central issue to be discussed here can be simply, if crudely, posed: Do the laws of physics allow the operation of a time machine, a device that produces CTCs? If the answer is negative and the reasons have to do with what happens before the CTCs are produced, then issues surrounding the paradoxes of time travel do not have to be

\footnotetext{
1 There are also various works on time travel and time machines that lie somewhere in between the popular science literature and the technical physics literature; see, for example, Hawking et al. (2002) and Nahin (1999).

2 A notable exception to our negative diagnosis of the philosophical literature is Arntzenius and Maudlin (2005).

3 Our complaint is not that a "functional" definition is lacking-a TM is a device that produces CTCsbut rather that there is not a standard definition of a TM in terms of spacetime structure, where the latter definition is needed to prove no-go theorems. Our thanks to an anonymous referee for prompting this clarification.
} 
confronted. But any attempt to give a positive answer cannot avoid taking a stance on these paradoxes. Our stance, as explained in Sect. 2, is that these paradoxes are not genuine antinomies but simply counterintuitive results that do not threaten either the conceptual coherency or the physical possibility of time travel. At the same time we try to diagnose why the air of paradox is so difficult to dispel. Section 3 surveys attempts to give necessary and/or sufficient conditions for the operation of time machines within the setting of relativistic spacetimes. We reject various key conditions that appear frequently in the physics literature, and we show why it is so difficult to specify an interesting sense in which a device operating in a relativistic spacetime can be said to be responsible for the development of CTCs. Although the most straightforward way of cashing out "responsible for" is blocked, we introduce a "potency condition" that we argue is the next best thing. The analysis in Sect. 3 deals with how such responsibility can be cashed out exclusively in terms of the causal structure of spacetime. The consequences of specific dynamical laws are not considered until later sections, and various spacetimes are considered in Sect. 3 that may not qualify as physically possible. But an analysis at this general level has the distinct merit of being more fundamental and thus applicable to all the different theories with all the different dynamics discussed in the remaining sections of the paper. In the light of this discussion and the potency condition we review in Sects. 4-7 purported no-go results for time machines, as well as more general "chronology protection" results, that come from the classical general theory of relativity (GTR), semi-classical quantum gravity, quantum field theory on curved spacetime, and Euclidean quantum gravity.

Our discussion of these results is motivated by two more general philosophical concerns, beyond their bearing on the question at hand. The first is that delimiting the content of a theory involves judgments regarding what qualifies as "physically reasonable," and the status of such judgments is a more interesting issue than philosophers have generally realized. In this case, the question is whether the vast array of spacetime structures allowed in GTR can be trimmed down by considering solutions that correspond to "reasonable" choices of physical fields populating spacetimes. We explore various ways of cashing out "reasonableness," from imposing energy conditions to formulating prerequisites a spacetime must satisfy to admit a quantum field theory (QFT). Our second motivation for considering these results is that they highlight the importance of "hybrid" theories and how they are employed. The no-go results reviewed in Sects. 5-7 all rely on different combinations of QFT and GTR, none of which constitute a fully satisfactory theory of quantum gravity. But physicists expect that exploring the no-go theorems will allow them to probe the reliability of different parts of each theory, and give some indication of what should carry over to a theory of quantum gravity. Our conclusions are presented in Sect. 8.

\section{Time travel and its paradoxes}

We will not review here the various different senses of "time travel." 4 And we will ignore those versions of time travel that exploit abnormal worldlines in a spacetime

\footnotetext{
${ }^{4}$ See Earman (1995) and Earman and Wüthrich (2004) for taxonomies and further discussion.
} 
that possesses a bland causal structure, such as Newtonian or Minkowski spacetime. By an "abnormal worldline" we mean, for example, a discontinuous world line that represents an observer who pops out of existence in 2008 and then pops back into existence in 1492, or a continuous worldline that bends backwards in time. ${ }^{5}$ We will be concerned exclusively with the sense of time travel that uses worldlines that do not involve either of these pathologies but which, nevertheless, are able to close on themselves because they reside in a spacetime with an abnormal causal structure.

In more detail, we will be concerned with relativistic spacetimes, which by definition consist of a differentiable manifold $\mathcal{M}$ and a Lorentz signature metric $g_{a b}$ defined on all of $\mathcal{M}$. We are interested only in cases where the spacetime $\mathcal{M}, g_{a b}$ is time orientable in the sense that it can be assigned a globally consistent time direction. (Technically, this means that the spacetime admits a continuous non-vanishing timelike vector field, implying that there is a continuous division of the lobes of the null cones into "future lobe" and "past lobe." This is a mild restriction since it can always be satisfied by passing, if necessary, to a covering spacetime, as follows from the fact that any simply connected spacetime is time orientable. Deciding which direction of a time oriented spacetime deserves to be labeled "future" and which deserves to be labeled "past" is part of the problem of the direction of time, which we do not try to tackle here.) By a wordline we mean a continuous timelike curve which at each point is assigned a temporal orientation that agrees with the time orientation of the spacetime in which it is embedded. A CTC is then a closed worldline.

We are concerned here only with senses of time travel that can be explicated using CTCs. The fans of science fiction stories on time travel may complain that our sense of time travel is a cheat since it implies ipso facto that there is no normal time in which the time traveler can "go backward," for CTCs exist only for spacetimes that possess a globally consistent time direction but not a globally consistent time order. ${ }^{6}$ They may be able to introduce some other notion of "time" such that travelling backwards makes sense in special cases, such as asymptotically flat spacetimes. (These spacetimes approach flat Minkowski spacetime "far away" from an isolated gravitational source. The lack of a globally consistent time order does not rule out the existence of an "asymptotic time" defined in terms of the additional structure of the asymptotic region. ${ }^{7}$ ) However, this strategy does not work in general, and we will not discuss such time travel scenarios further. We acknowledge this limitation of our approach,

\footnotetext{
5 Of course, the problem is to make sense of the "and then" and "bends backwards."

${ }^{6}$ For $p, q \in \mathcal{M}, g_{a b}$ define $p \ll q$ ( $p$ chronologically precedes $q$ ) to hold iff there is a future directed timelike curve from $p$ to $q$. This relation is always transitive. But it will be irreflexive and anti-symmetric iff the spacetime does not contain CTCs.

7 As an example of how a time travel scenario might work in such cases, consider a Deutsch-Politzer gate, as illustrated in Fig. 5a, assuming that this acausal region can be embedded in an asymptotically flat spacetime. Our would-be time traveler could enter the gate "just before it closes" (the hypersurface connecting $p_{1}$ with $p_{3}$ ) and emerge from the "opening" of the gate (the hypersurface connecting $p_{2}$ with $p_{4}$ ) at an earlier (asymptotic) time. Even in this example it is impossible to access asymptotic times prior to the formation of the acausal region, ruling out some time travel scenarios. Furthermore, "topological censorship" theorems due to Friedman, Schleich and Witt (1993) indicate that (given various assumptions) any timelike curve that threads a region of complicated topology cannot return to the asymptotic region; if these theorems can be extended to non-globally hyperbolic spacetimes, they would rule out such a time travel scenario.
} 
but we note that the trade-off for the loss of contact with the science fiction literature is a gain in contact with the theories of modern physics.

In any case, for us the question "Is time travel possible?" is equivalent to the question of whether a spacetime structure containing CTCs is physically possible. This issue can be given two senses, corresponding to a weak and a strong sense of physical possibility. Time travel will be said to be physically possible in the weak sense just in case the laws $L$ (of the actual world) are such that CTCs are compatible with $L$, or, in possible worlds talk, just in case there is a possible world in which there are CTCs and in which $L$ are true. Time travel will be said to be physically possible in the strong sense just in case the laws $L$ (of the actual world) are such that there is a possible world in which there are CTCs and in which $L$ are not only true but are laws. If the laws $L$ are the laws of Einstein's GTR, then time travel is physically possible in the weak sense, for there are many known solutions to Einstein's field equations which satisfy various energy conditions, ${ }^{8}$ thought to be necessary for physically realistic matter-energy sources, and which contain CTCs.

The issue of physical possibility in the strong sense is more delicate and controversial because it turns on one's conception of laws of nature. If, for example, one wants an empiricist conception of laws on which the laws supervene on the occurrent regularities, then the weak sense of physical possibility does not entail the strong sense since lawhood is not necessarily transmitted down the chain of nomological accessibility. Consider, for sake of illustration, David Lewis's (1973) "best systems analysis" of laws, ${ }^{9}$ and suppose that the best system for the actual world is axiomatized by the postulates of GTR. There is no guarantee that these postulates will be among the axioms or theorems of the best system for a possible world that contains CTCs and that satisfies these postulates. Nevertheless, we would be very surprised if there were not some possible worlds that correspond to solutions to Einstein's field equations, that contain CTCs, and that return the laws of GTR when the best systems analysis is applied to them. On other analyses of laws, such as Carroll's (1994), the present issue doesn't arise since the weak sense of physical possibility entails the strong sense. The price to be paid for obtaining this entailment is the failure of laws to be Humean supervenient, a price we think is too high; but that is a topic for another occasion. $^{10}$

If it is so clear that time travel is physically possible in the weak sense and (probably) also in the strong sense, ${ }^{11}$ what is one to make of the discussions in the philosophical literature on the paradoxes of time travel, which are often taken to threaten the conceptual coherency of time travel? The grandfather paradox is a staple of these

\footnotetext{
8 These energy conditions will be discussed below in Sect. 4.

9 Roughly, this view holds that the laws of a world $W$ are given by the axioms or theorems of the best deductive system for $W$. A deductive system for $W$ is a deductively closed axiomatizable set of sentences, all of which are true in $W$. The best such system is the one that achieves the best compromise between strength (or information content) and simplicity.

10 A good overview of the different currently available accounts of laws of nature is found in J. W. Carroll (2004).

11 At least as far as the laws of classical GTR are concerned. Adding quantum considerations may yield a different verdict. But the angst about the paradoxes of time travel that is evident in the philosophical literature does not derive from worry about an incompatibility of time travel with quantum physics.
} 


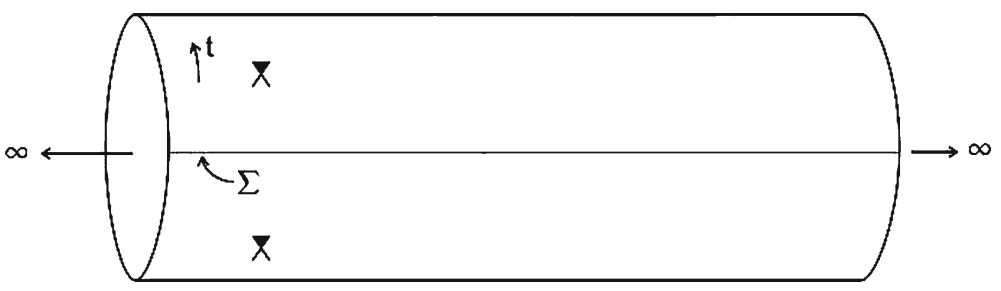

Fig. $1(1+1)$-dimensional Minkowski spacetime rolled up along the time axis

discussions-Kurt travels into his past, kills his grandfather before granddad sires Kurt's father, thus preventing Kurt from making the journey. We view such paradoxes as a way of calling attention to a phenomenon long known to physicists; namely, in the presence of CTCs, consistency constraints can arise for local physics that would be absent in spacetimes with a tame global causal structure. ${ }^{12}$ Familiar laws of relativistic physics are typically stated in terms of local differential equations, and as a result they have a global-to-local property. Treat a spacetime $\mathcal{M}, g_{a b}$ as a fixed background on which a field $\Phi$ propagates. (Here "field" is to be given a broad interpretation-it may be a scalar, vector, or tensor field, and it may describe a system of discrete particles as opposed to a continuum field.) The laws $L$ governing $\Phi$ have the intended globalto-local property iff for any global solution $\left\langle\mathcal{M}, g_{a b}, \Phi\right\rangle$ and any open neighborhood $\mathcal{U} \subset \mathcal{M},\left\langle\mathcal{U}, g_{a b}|\mathcal{U}, \Phi| \mathcal{U}\right\rangle$ is also a solution. If the causal structure of the background spacetime $\mathcal{M}, g_{a b}$ is tame one would expect that the field also has the opposite localto-global property that any local solution $\left\langle\mathcal{U}, g_{a b}|\mathcal{U}, \Phi| \mathcal{U}\right\rangle$ can be extended to a global solution $\left\langle\mathcal{M}, g_{a b}, \Phi\right\rangle$. But when CTCs pass through $\mathcal{U}$ (or more generally $\mathcal{U}$ is intersected repeatedly by a future directed timelike curve) this local-to-global property can fail. A trivial but instructive illustration is obtained by rolling up $(1+1)$-dimensional Minkowski spacetime along the time axis (see Fig. 1). Let $\mathcal{U}$ be a thin sandwich about the time slice $\Sigma$. And consider on $\mathcal{U}$ a solution to the relativistic wave equation for a scalar field $\Phi$ that corresponds to a single localized disturbance propagating to the right. ${ }^{13}$ This local solution does not extend to any global solution on the cylinder spacetime. If the spacetime of Fig. 1 is made into a donut by identifying points of the space axis that are equal modulo $\pi$, then the only local solutions of the wave equation on any open $\mathcal{U} \subset \mathcal{M}$ that can be extended to a global solution are the trivial ones, i.e. $\Phi=$ const ${ }^{14}$

Both the philosophical and physics literature contain assertions to the effect that there are no genuine paradoxes of time travel. If this is taken to mean that the grandfather paradox and its ilk do not demonstrate that time travel is logically, conceptually, or physically absurd, then we agree wholeheartedly. For, to repeat, such paradoxes

\footnotetext{
12 We should emphasize that we are interested only in consistency constraints entailed by the combination of local physical laws and global spacetime structure, and not with a broader notion of consistency (such as classifying initial data that lead to the formation of a singularity as somehow "inconsistent").

13 The wave equation states: $\nabla^{2} \Phi-\frac{\partial^{2} \Phi}{\partial t^{2}}=0$, where units have been chosen so that $c \equiv 1$.

14 It has been conjectured, but not proved, that a similar triviality result holds for Gödel spacetime, which has the property that there is a CTC through every point.
} 
simply illustrate the presence of consistency constraints on local physics in spacetimes with CTCs; and the presence of such constraints do not show that CTCs are physically impossible in either the strong or weak sense. But sometimes the assertion that there are no genuine paradoxes of time travel is taken to mean that non-trivial consistency constraints are absent (see, for example, Krasnikov 1997). In unqualified terms, such assertions are patently false, as illustrated not only by the above artificial example but by many other examples of self-interacting and non-self-interacting fields on a variety of general relativistic spacetimes with CTCs. There is, however, a qualified sense in which this assertion points to an interesting truth.

Suppose that a spacetime $\mathcal{M}, g_{a b}$ contains a global time slice $\Sigma$ (i.e., a spacelike hypersurface with no edges). ${ }^{15}$ Irrespective of the global causal structure of $\mathcal{M}, g_{a b}$, the initial data on $\Sigma$ for a relativistic field $\Phi$ may not be freely specifiable but must satisfy a set of constraints $\mathcal{C}$ that follow from the laws $L(\Phi)$ governing $\Phi$. For example, the source-free Maxwell equations for the electromagnetic field entail that the electric and magnetic fields at "time" $\Sigma$ are divergence free; similarly, initial data for Einstein's field equations must satisfy a pair (or triple) of constraint equations. If $\Sigma$ is intersected more than once by future directed timelike curves, then consistency with $L(\Phi)$ will typically entail additional constraints on initial data over and above $\mathcal{C}$. But suppose that $\Sigma$ is achronal (i.e. is not intersected more than once by a future directed timelike curve), in which case $\Sigma$ is called a partial Cauchy surface. ${ }^{16}$ Does the presence of a non-empty chronology violating region $\mathcal{V} \subset \mathcal{M}$ (consisting of all points $p \in \mathcal{M}$ such that a CTC passes through $p)^{17}$ not intersecting $\Sigma$ give rise to consistency constraints over and above $\mathcal{C}$ in the sense that it is not true that, given any initial data on $\Sigma$ satisfying $\mathcal{C}$, there is at least one solution of $L(\Phi)$ on all of $\mathcal{M}, g_{a b}$ that agrees with the given data set on $\Sigma ?^{18}$ For fundamental fields-those which admit a well-posed global initial value problem in Minkowski spacetime ${ }^{19}$-no counterexamples are known to the conjecture that additional consistency constraints do not arise. And a result of Krasnikov (2002), which will be discussed below, can perhaps be adapted to prove this conjecture for a broad range of cases.

For future reference, however, note that a quantum mechanical treatment of this issue may yield a different conclusion; indeed, there are a number of results to the effect that for interacting fields, unitarity fails for the chronology violating region $\mathcal{V}$ to the future (or past) of $\Sigma$ (see Boulware 1992; Friedman et al. 1992; Politzer 1992). There are various proposals for securing unitarity (see Deutsch 1991; Deutsch and Lockwood 1994; Fewster and Wells 1995), but the security comes with a price tag.

\footnotetext{
15 Some spacetimes, such as Gödel spacetime, do not admit any global time slices. This is a consequence of three features: it is time orientable; a CTC passes through each point; and it is simply connected. The edge of an achronal surface $S$ is the set of points $p$ such that every open neighborhood $O \ni p$ includes points in $I^{+}(p)$ and $I^{-}(p)$ that can be connected by a timelike curve that does not cross $S$.

16 If $\Sigma$ is a global time slice of a spacetime $\mathcal{M}, g_{a b}$ then it will become a partial Cauchy surface in an appropriate covering space time. For example, the time slice $\Sigma$ of the spacetime of Fig. 1 becomes a partial Cauchy surface when this spacetime is "unrolled" to become Minkowski spacetime.

17 If $\mathcal{V} \neq \varnothing$ then it is an open region.

18 Typically, if there is one such solution there will be many since the chronology violation region $\mathcal{V}$ will lie beyond the domain of dependence of $\Sigma$, as discussed below.

19 These include the Klein-Gordon field and the source-free Maxwell field.
} 
For example, Deutsch's treatment uses the many worlds interpretation of quantum mechanics plus the assignment of mixed rather than pure states to individual systems. We will see below that quantum considerations provide the best hope for no-go result for time machines.

Anticipating the discussion in the following section, our characterization of time machines implies that there is a partial Cauchy surface on which the operating instructions for the machine are specified. Thus, insofar as the paradoxes of time travel are concerned with consistency constraints, "no-go" results for time machines that depend only on what happens before the formation of CTCs are independent of the paradoxes. However, a purported "go" result would be tainted by the paradoxes-if they are indeed paradoxes, rather than results that indicate a need to retrain our intuitions which are tutored on cases without pathologies due to CTCs.

A diagnosis of one source of the lingering air of paradox can be gleaned from the following quotation from a recent article in Physical Review D, a leading physics journal:

Satisfactory physical theories must avoid giving rise to ... self-contradictory predictions. One approach to achieving this is to impose consistency constraints on the allowable initial conditions on spacelike surfaces prior to the formation of the CTCs, thus abandoning the principle that initial conditions on such surfaces can be chosen at will. For example, in the case of the grandfather paradox we might insist that the initial conditions just before the prospective murder include the presence of a strategically placed banana peel on which the prospective murderer slips as he pulls the trigger, thus spoiling his aim. One might refer to this approach as the 'banana peel mechanism'... (Everett 2004, p. 124023-1)

As the quotation indicates, the feeling of paradox arises from the conflict between the notion that initial conditions ought to be freely specifiable and the idea that highly contrived "banana peel mechanisms" have to be imposed to avoid a self-contradictory result in the presence of CTCs. ${ }^{20}$

We advocate dispelling the air of paradox as follows. First, the notion that initial conditions should be freely specifiable is simply wrong-as noted above, even in the absence of global causal pathologies relativistic field equations may impose non-trivial constraints $\mathcal{C}$ on initial data. As for the additional constraints that arise when CTCs are present, no Cosmic Chaperone is needed to make sure that they are in place, for these additional constraints are entailed by the conjunction of the local laws governing the fields and the global structure of the spacetime on which the fields are propagating. This entailment arguably gives the additional constraints a lawlike status and, as such, they arguably require no more explanation than do the constraints $\mathcal{C}$. A caveat is needed because there are distinctions between familiar constraints and the constraints due to CTCs, emphasized by Arntzenius and Maudlin (2005): the constraints imposed on data specified on a surface $\Sigma$ due to the presence of CTCs depends on the global structure of the spacetime and how $\Sigma$ is embedded within it. Although we will not

\footnotetext{
20 This point has also been noted in the philosophical literature, and the need for "banana peel mechanisms" has been used to argue for the improbability of time travel; see, e.g., Horwich (1987), and our response below.
} 
argue the point here, our position is that these differences do not undermine our claim that the "banana peel mechanisms" have the lawlike force of constraints. We endorse the deflationary option that Everett offers when he concedes that the feeling that initial conditions are freely specifiable and that banana peel mechanisms are needed to avoid paradoxes "may simply reflect our lack of experience with phenomena involving CTCs" (ibid.).

This discussion reveals a second, closely related source of the air of paradox about time travel; namely, since we typically assume a tame global spacetime structure, we naturally explicate the notion of what is physically possible locally in time or spacetime as the compatibility of a local state of affairs with the local laws of physics. That $X$ is locally physically possible in this sense normally guarantees that $X$ is physically possible in-the-large, in that $X$ can be realized as part of a global scenario compatible with the same laws. But when the background spacetime contains a $\mathcal{V} \neq \varnothing$ the guarantee can fail, since the local-to-global property described above may fail. When the guarantee does fail one has the choice of modifying the explication of local physical possibility to include a requirement of global realizability, or else of canceling the expectation that being locally physically possible entails global realizability. Taking spacetimes with CTCs seriously requires this "globalization" of physical possibility, and the grandfather paradox and the like serve to highlight this surprising consequence. It is (almost) universally agreed that nothing illuminating comes from trying to promote the clash between the special theory of relativity and intuitions about space and time that have been tutored by Newtonian physics into "paradoxes of relativity," save when these "paradoxes" are used as a pedagogical device to highlight some of the differences between the old and the new theory. Our analysis of the time travel paradoxes is similar: they highlight the contrast between the analysis of physical possibility in a spacetime with tame global structure and in a setting with CTCs.

Perhaps the paradoxes reveal something else entirely, namely that CTCs are implausible or unlikely even if they are not logically or conceptually incoherent? This response grants our argument above, but then infers the improbability of CTCs from the "bizarre" nature of such constraints (see, e.g, Arntzenius and Maudlin 2005). The image of Kurt repeatedly slipping on a banana peel as he tries to shoot Grandpa suggest that the constraints would be strong enough to be immediately at odds with intuitive judgments of local physical possibility. It is not yet known what form these constraints will take for anything beyond simple toy models, and so it is unclear how much we would be forced to revise our judgments regarding physical possibility. Although we do not have space to pursue the issue fully here, we see two problems with arguments based on the "bizarre" nature of the constraints. First, the constraints are relevantly similar to other constraints that are a familiar and well-understood, if often overlooked, feature of relativistic field equations. Pre-Maxwell physicists may have had intuitions regarding the assignments of electric and magnetic fields that conflicted with the constraint equations, but in what sense would noting their "bizarre-ness" relative to pre-theoretical intuitions be a revealing criticism of Maxwell's theory? Second, it is unclear what is meant by the claim that CTCs are "improbable." This could mean that the "best fit" model of GTR that describes that universe or a subsystem of it probably does not include CTCs. It is not clear how the existence of constraints would be relevant to assessing this probability, or that the "bizarre nature" of constraints would trump 
other considerations. On the other hand, the claim could instead be read as a negative assessment of the subjective probability or degree of belief for the theory-namely, that any theory allowing CTCs and bizarre constraints on initial conditions should be assigned a low probability. Something like this second sense of "improbability" may motivate physicists pursuing no-go theorems. But even if that is the case there are other more compelling reasons to assign a low probability to classical GTR, e.g., that it is incompatible with quantum theory.

Our deflationary resolution of the paradoxes of time travel has relied on treating a spacetime with a chronology violating region as a fixed background on which test fields propagate. This treatment involves an idealization that may be legitimate for some purposes, but strictly speaking it is inconsistent with both the letter and spirit of GTR, which implies that the structure of spacetime is not fixed but co-evolves with the matter-energy content according to the coupled Einstein-matter field equations. It is, therefore, reasonable to request that the above discussion be redone with the idealization removed. Our response is to forthrightly admit that we cannot fulfill the request, and then to say so much the worse for the paradoxes of time travel! The point is that without the idealization that treats a spacetime with a chronology violating region as fixed background, it is difficult to make sense of the notion of the consistency constraints that flow from the presence of CTCs. What one wants to know is the answer to: What initial conditions for the coupled Einstein-matter field equations, satisfying the constraints $\mathcal{C}$ appropriate for these coupled equations, can be realized in a spacetime containing a chronology violating region $\mathcal{V} \neq \varnothing$ and displaying features

? Different ways of filling the blank can produce radically different answers. The ambiguity is removed when one works with a fixed spacetime background; for then the filling of the blank is automatically specified, and the answer to the question at issue is entailed by the character of the solution set for the coupled Einstein-matter field equations on the given background spacetime. But when the fixed background is removed, there seems to be no principled way to fill the blank, and the choice of filling is at the whim of pragmatic factors, such as the type of time travel story one happens to be interested in at the moment. Thus, to the extent that the legitimate interest of the so-called paradoxes of time travel is captured by the issue of consistency constraints, that interest drains away as the notion of consistency constraints turns to mush, as it seems to when the idealization of a fixed spacetime background is removed.

\section{Operating a time machine}

That time travel is physically possible-in the sense that there are physically possible worlds (in either the weak or strong sense) with CTCs-does not necessarily mean that it is physically possible to operate a time machine. Our goal in this section is to get a grip on what the operation of a time machine would mean in terms of relativistic spacetime structure, over and above the existence of CTCs.

Before starting on this task in earnest we should briefly explain our approach and respond to a potential objection. Our aim is to clarify the sense in which operation of a time machine can be said to "cause" or "produce" CTCs. The nature of the problem disbars straightforward proposals, e.g. that the specification of initial data for physical 
fields within a TM region "produces" CTCs via dynamical evolution. This idea fails because the CTCs necessarily fall outside the region of spacetime fixed via dynamical evolution from the TM region, as we spell out below. In its place we propose a Potency Condition, based on the intuition that although CTCs cannot lie within the region causally determined by the TM region, they may be on the verge of forming - in the sense that every possible extension of the TM region contains CTCs. Throughout this discussion we characterize "causation" in terms of spacetime structure. But perhaps this focus is too narrow, and there is some perfectly legitimate sense in which a time machine "causes" the formation of CTCs that our analysis doesn't capture? To this objection our response is simply to encourage the objector to explain this broader notion of "cause" that we have failed to capture, and warn her of the challenge she faces. We suspect that analyses of causation developed to handle ordinary cases of causation will not be of much use in considering time machines. Absent some broader notion of causation applicable to this case, we proceed to use the technical tools that do shed light on the nature of time machines.

Another aspect of our approach deserves comment: in this section we give a definition of a TM, delaying the assessment of physical possibility until later sections. The concepts of causal structure employed in the definition are both more general and more fundamental than a particular choice of field equations. They are a common ingredient of all the hybrids of quantum theory and GTR discussed below, so our definition can be employed across this range of cases. Admittedly, quantum gravity may someday reveal these ideas are only approximations to some quite different underlying structure, but that day is not yet here-so we continue to rely on them. Once we have a grasp on the spacetime geometry of a TM, we consider whether such a geometrical configuration falls within the space of physical possibilities delimited by a given set of field equations.

Returning to our task, if the spacetime $\mathcal{M}, g_{a b}$ is to accommodate a time machine then it should be possible to choose a global slice $\Sigma$, corresponding to a time before the time machine goes into operation, such that no CTCs exist up to "time" $\Sigma$; for otherwise there would be no need for a time machine. (Thus, Gödel spacetime, so beloved by philosophers who write on time travel, is ruled out in the first instance as a time machine spacetime since it lacks a global slice.) Without much loss of generality, it can be assumed that $\Sigma$ is a partial Cauchy surface. ${ }^{21}$ Then the conditions that encode the instructions for the operation of the time machine can be set on $\Sigma$. To assure that the instructions are obeyed, the region of spacetime $\mathcal{T} \mathcal{M} \subset \mathcal{M}$ corresponding to the operation of the time machine should belong to the future domain of dependence $D^{+}(\Sigma)$ of $\Sigma$. $\left(D^{+}(\Sigma)\right.$ consists of all points $p \in \mathcal{M}$ such that every past intextendible causal curve through $p$ intersects $\Sigma$. The past domain of dependence $D^{-}(\Sigma)$ is defined analogously. $\Sigma \subset \mathcal{M}$ is dubbed a Cauchy surface if its total domain of dependence $D(\Sigma):=D^{+}(\Sigma) \cup D^{-}(\Sigma)$ is $\mathcal{M}$. A spacetime that admits a Cauchy surface is said to be globally hyperbolic. ${ }^{22}$ ) And whatever the details of its operation, a time machine is

\footnotetext{
21 Recall that if $\Sigma$ is a global time slice for $\mathcal{M}, g_{a b}$, then $\Sigma$ can be turned into a partial Cauchy surface by passing (if necessary) to a covering spacetime of $\mathcal{M}, g_{a b}$.

22 For the official definition of global hyperbolicity and a proof that a spacetime is globally hyperbolic iff it admits a Cauchy surface, see Hawking and Ellis (1973, pp. 206-212). For the hyperbolic field equations
} 


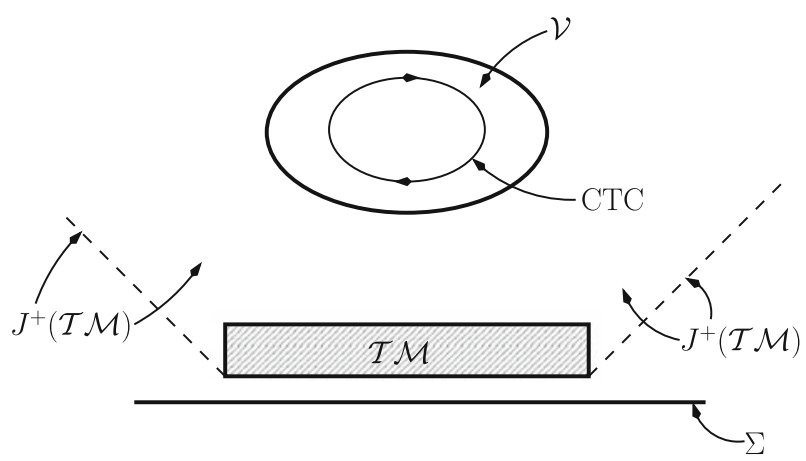

Fig. 2 A spacetime containing a would-be time machine

supposed to be confined to a finite region of space and to operate for a finite amount of time, implying that $\mathcal{T} \mathcal{M}$ should have compact closure. ${ }^{23}$ And finally, to help capture the idea that CTCs result from the operation of the time machine, it should be the case that the chronology violating region $\mathcal{V}$ is contained in the causal future $J^{+}(\mathcal{T} \mathcal{M})$ of $\mathcal{T} \mathcal{M}$. $\left(J^{+}(\mathcal{S}), \mathcal{S} \subset \mathcal{M}\right.$, is defined as the set of all $p \in \mathcal{M}$ such that $p$ can be reached from $\mathcal{S}$ via a future directed causal curve. The causal past $J^{-}(\mathcal{S})$ of a set $\mathcal{S}$ is defined analogously. $)^{24}$ These requirements are summarized schematically in Fig. 2.

This is very sketchy, but even without filling in the details two difficulties should be apparent. The first is that no matter how this scenario is filled in, it will not yield the kind of time travel beloved by science fiction writers, for traveling from the "time" $\Sigma$ into the causal past $J^{-}(\Sigma)$ of $\Sigma$ is ruled out by the set-up. The kind of time travel promoted by a time machine is thus confined to the future of the "time" $\Sigma$. We see no way around this limitation as long as time machines are to be implemented in orthodox relativistic spacetimes.

A second and much more serious difficulty lies in making sense of the notion that CTCs are the "result of" the operation of the time machine. This notion cannot be understood in the sense of causal determinism since $\mathcal{V}$, if it is non-null, always lies outside $D^{+}(\mathcal{T} \mathcal{M})$ and, indeed, outside of $D^{+}(\Sigma)$. Nor does it help to point to the stipulation that $\mathcal{V} \subset J^{+}(\mathcal{T} \mathcal{M})$, for while that condition guarantees that every point in the chronology violating region region can be causally influenced by the operation of the time machine, it does not rule out the possibility that $\mathcal{V}$ is affected, perhaps decisively, by influences not emanating from $\mathcal{T} \mathcal{M}$. Requiring that $J^{-}(\mathcal{V}) \cap \Sigma$ has compact closure guarantees that any influences on the time travel region emanating from $\Sigma$ must originate from a finite portion thereof, which is certainly desirable since conditions on

Footnote 22 continued

encountered in relativistic theories, there are typically existence and uniqueness theorems that say in effect that appropriate initial conditions on a partial Cauchy surface $\Sigma$ uniquely determine a solution throughout the globally hyperbolic region $D(\Sigma)$.

23 This rules out seeing Gott's (1991) model as a time machine since it uses two infinitely long cosmic strings.

24 Thus, it is somewhat misleading to characterize no-go results as making "the universe safe for historians" (Hawking 1992a, p. 603) since the very set-up of the problem automatically gives this safety. 


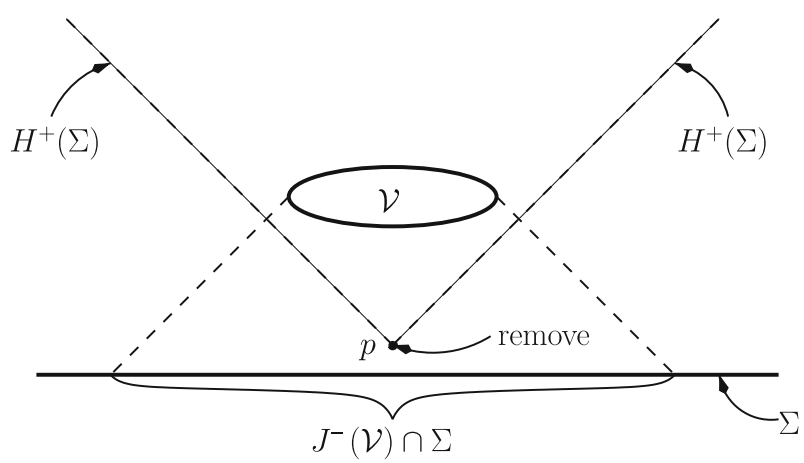

Fig. 3 Influences coming from a singularity or from infinity

$\Sigma$ specify the operation of the time machine and such a device is supposed to be finite. But again the trouble is that the condition in question does not ensure that there aren't other influences, perhaps decisive, on $\mathcal{V}$ which originate from outside $J^{-}(\mathcal{V}) \cap \Sigma$ or even from outside of all of $\Sigma$. The worry is illustrated by the simple but contrived example of Fig. 3. The missing point $p$ represents a singularity from which causal influences, not determined by the conditions on $\Sigma$, can emerge and propagate to effect $\mathcal{V}$, even though $J^{-}(\mathcal{V}) \cap \Sigma$ has compact closure. This trivial singularity can be made irremovable, without changing the causal structure pictured in Fig. 3, by replacing the original metric $g_{a b}$ by $\phi^{2} g_{a b}$ where $\phi$ is a scalar field that goes to zero as the missing point is approached. Alternatively, if $\phi$ is chosen to "blow up" as the missing point is approached, the singularity can be made to disappear by pushing it off to infinity. But in that case $\mathcal{V}$ is subject to influences coming from "infinity."

Hawking (1992a) presumably had in mind these sorts of difficulties when he imposed the requirement that the future Cauchy horizon $\mathrm{H}^{+}(\Sigma)$ of $\Sigma$ be compactly generated (see also Hawking 1992b). $H^{+}(\Sigma)$ is the future boundary of $D^{+}(\Sigma)$ (i.e. $\overline{D^{+}(\Sigma)}-I^{-}\left(D^{+}(\Sigma)\right)$, where the overbar denotes topological closure and $I^{-}(\mathcal{S})$, $\mathcal{S} \subset \mathcal{M}$, is the chronological past of $\mathcal{S}$ consisting of all points $p$ such that there is a past directed timelike curve from $\mathcal{S}$ to $p$ ). $H^{+}(\Sigma)$, which bounds the part of spacetime whose state can be causally determined by conditions on $\Sigma$, is necessarily a null surface, and its generators are null geodesics. If these generators, when traced far enough into the past, fall into and remain in a compact set, then the Cauchy horizon is said to be compactly generated. This requirement precludes the possibility of the generators emerging from a curvature singularity or coming from infinity and, thus, rules out the example of Fig. 3, whether or not it is doctored by the addition of a conformal factor; for no neighborhood of the missing point $p$ has compact closure, and thus every past directed generator of $H^{+}(\Sigma)$ will not remain trapped within a compact set.

It is not clear at the outset whether Hawking's condition is either necessary or sufficient for the operation of a time machine. As for necessity, it is true that a violation of Hawking's condition opens the possibility that the chronology violating region is affected by influences coming not from the would-be time machine but from a 
singularity or from infinity; but it does not follow that this is in fact the case. ${ }^{25}$ And as for sufficiency, it is not at all obvious how, even in conjunction with the other restrictions imposed above, the requirement that the chronology violating region is not affected by influences coming from infinity or singularities ensures that the development of CTCs is "due to" the operation of the would-be time machine.

One obvious and seemingly attractive way to obtain such an assurance is to require that every suitable extension of $D^{+}(\Sigma)$ contains CTCs-call this the Potency Condition for the would-be time machine. ${ }^{26}$ Satisfaction of the Potency Condition would not guarantee that the details of the particular way in which CTCs in fact developed are due to the operation of the would-be time machine; but we know already that no condition can provide such a guarantee, at least not via causal determinism. Satisfaction of the Potency Condition would provide the next-best-thing by guaranteeing the development of CTCs in some form or other. Of course, the condition as stated remains a schema rather than a definite condition until "suitable extension" is defined. At a minimum the extension should be smooth and maximal (i.e. does not admit a proper extension). At the very least, any dynamical theory will add as a condition the satisfaction of its dynamical equations. For reasons given above, we do not impose any of these conditions just yet although we recognize that ultimately, dynamical conditions must be included in an assessment of physical possibility. We will get to the fate of time machines in various dynamical theories in Sects. 4-7. Here, we attempt to lay bare the most fundamental causal spacetime structure that they all share. In general, the more additional restrictions that are put on the allowed extensions, the weaker the potency.

There is little hope, however, that the strongest form of the Potency Condition is ever realized. To see the reason for the pessimism, consider the case of Misner spacetime (see Fig. 4a), a two-dimensional spacetime which captures some of the causal features of Taub-NUT spacetime, itself a homogeneous vacuum solution to Einstein's field equations. Misner spacetime is topologically $S^{1}$ x $\mathbb{R}$. In the lower, or Taub region, the surfaces of homogeneity, such as $\Sigma$, are spacelike. But because the light cones "tip over," the surfaces of homogeneity eventually become more and more lightlike, until eventually one reaches $H^{+}(\Sigma)$, which is not only compactly generated but compact. If the Potency Condition is going to be satisfied in a strong form, Misner spacetime would seem to be an ideal candidate; for $\mathrm{H}^{+}(\Sigma)$ contains a closed null curve (CNC), indicating that CTCs are on the verge of forming. But not every smooth maximal extension of the $D^{+}(\Sigma)$ contains CTCs; nor does every such extension have a compact or compactly generated $\mathrm{H}^{+}(\Sigma)$ containing CNCs. A smooth maximal extension sans CTCs and sans compactly generated $H^{+}(\Sigma)$ can be constructed by cutting out vertical strips as shown in Fig. $4 \mathrm{~b}$ and slapping on appropriate conformal factors that preserve the causal structure and ensure maximality by "blowing up" as the cut-out regions are approached. Requiring that "suitable extensions" satisfy Einstein's field

\footnotetext{
25 As was pointed out to us by Amos Ori.

26 Or even more stringently that every suitable extension of $D^{+}\left(\Sigma_{O}\right)$, for some compact $\Sigma_{O} \subset \Sigma$, contains CTCs.
} 

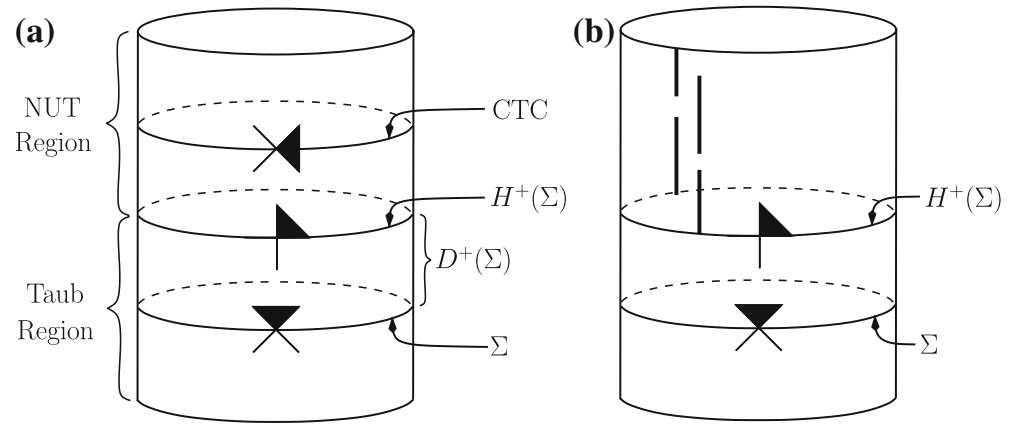

Fig. 4 (a) Misner spacetime, (b) Misner spacetime with strips removed

equations and standard energy conditions ${ }^{27}$ would presumably disbar such constructions but would also, of course, weaken the potency. ${ }^{28}$

However, even if the surgery-and-conformal-factor trick is disbarred, a CTC-free maximal extension of the Taub region can still be constructed by the alternative method of blocking CTC formation by first cutting out vertical strips (as in Fig. 4b) and then ensuring maximality by taking the universal covering spacetime of the surgically mutilated spacetime. ${ }^{29,30}$ To disbar such artifices in a non-question begging fashion and to give the Potency Condition a fighting chance of being instantiated, we will impose Geroch's (1977) condition of hole freeness. A spacetime $\mathcal{M}, g_{a b}$ is said to be hole free just in case for any spacelike $\Sigma \subset \mathcal{M}$ (not necessarily a global time slice) there is no isometric imbedding $\iota: D(\Sigma) \rightarrow \mathcal{M}^{\prime}$ into a spacetime $\mathcal{M}^{\prime}, g_{a b}^{\prime}$ such that $\iota(D(\Sigma))$ is a proper subset of $D(\iota(\Sigma)) .{ }^{31}$ That it is not question begging to require hole freeness in the present context follows from two considerations. First, we are seeking, so to

\footnotetext{
27 These conditions, which will be discussed in the following section, rule out negative energy densities and the like.

${ }^{28}$ Recall that our goal here is to offer a definition of a TM in terms of spacetime geometry without assessing physical possiblity. Within the context of a particular theory, such as classical GTR, it is natural to require that the extensions satisfy Einstein's field equations and various energy conditions, and then attempt to prove no-go theorems; see Sect. 4.

${ }^{29}$ We are indebted to Robert Geroch for this example, as well as for the suggestion to use the condition of hole freeness (see below), which was first introduced in Geroch (1977).

30 Ori (2005) has described a time machine model for which he claims that every regular extension beyond the Cauchy horizon includes a chronology violating region. The claim seems plausible-if the sorts of tricks we are discussing are disbarred. Ori (2007) appeals to a condition similar to our Potency Condition in order to capture the notion that the formation of CTCs is "triggered by" the initial conditions on (see 044022-3). Our Potency Condition was originally proposed in the pre-print "Take a Ride on a Time Machine" posted to the PhilSciArchive in 2003 and in revised form on 19 March 2004 (see philsciarchive.pitt.edu/archive/00001673). Since then this condition has appeared in various guises in the physics literature on time machines.

${ }^{31}$ In defining the domain of dependence $D(\Sigma)$ of $\Sigma$ it is typically assumed (if only implicitly) that $\Sigma$ is achronal. Since we are applying the notion of hole freeness to possibly acausal spacetimes, we cannot make this assumption. Thus, $D(\Sigma)$ is to be understood to consist of all points $p$ such that every inextendible causal curve through $p$ meets $\Sigma$ at least once (and possibly many times). As far as we can tell, this understanding leads to no counterintuitive consequences. But if this is incorrect, the definition of hole freeness can be modified as follows: $\mathcal{M}, g_{a b}$ is counted as hole free just in case for any open $\mathcal{U} \subset \mathcal{M}$ and any achronal
} 
speak, the next best thing to the notion that the operation of the would-be time machine causally determines that CTCs emerge. And, second, causal determinism itself has no prayer of being satisfied unless hole freeness is required (see Earman 1995, Sec. 3.8).

With this understanding, our proposed version of the strongest form of the Potency Condition requires that every extension of $D^{+}(\Sigma)$ contains CTCs if it is smooth and hole free. Manchak (2007) has very recently proven that Misner spacetime satisfies a slightly modified version of the strongest Potency Condition in which it is required that the closure of $D^{+}(\Sigma)$ be hole free. ${ }^{32}$ If the class of spacetimes satisfying this form of the condition should prove to be empty with the exception of Misner spacetime, the potency can be weakened by requiring in addition that extensions satisfy Einstein's field equations and energy conditions, or field equations derived from a proposed theory of quantum gravity. Thus, a hierarchy of Potency Conditions of decreasing strength emerges, indexed according to the theory used to delimit the physically possible extensions, with a correspondingly growing class of spacetimes satisfying the conditions.

To see the bite of the Potency Condition, consider a proposal of Krasnikov (1998) for defining relativistic time machines.

Definition Let $\mathcal{M}, g_{a b}$ be an inextendible spacetime with a non-null chronology violating region $\mathcal{V} . \mathcal{K} \subset \mathcal{M}$ is said to be a Krasnikov time machine iff (i) $\mathcal{V} \subset \mathcal{K}$ and (ii) $\mathcal{M}-J^{+}(\mathcal{K})$ is isometric to a region $\mathcal{M}^{\prime}-J^{+}\left(\mathcal{K}^{\prime}\right)$ of a spacetime $\mathcal{M}^{\prime}, g_{a b}^{\prime}$ that does not contain CTCs and $\mathcal{K}^{\prime} \subset \mathcal{M}^{\prime}$ is compact.

Footnote 31 continued

spacelike $\Sigma$ of the spacetime $\mathcal{U}, g_{a b} \mid \mathcal{U}$ there is no isometric imbedding $\iota: D(\Sigma) \rightarrow \mathcal{U}^{\prime}$ into a spacetime $\mathcal{U}^{\prime}, g_{a b}^{\prime}$ such that $\iota(\Sigma)$ is achronal and $\iota(D(\Sigma))$ is a proper subset of $D(\iota(\Sigma))$.

32 In fact, he prefers a slightly modified definition of "future domain of dependence" according to which $D^{+}(\Sigma)$ consists of all points in $\mathcal{M}$ from which every past-directed, inextendible timelike curve intersects $\Sigma$. In this case, he shows, Misner spacetime satisfies the Potency Condition even without imposing hole freeness. The extensions must only be maximal and smooth. The standard definition of $D^{+}(\Sigma)$ which we use here, can be found in Hawking and Ellis (1973, p. 201) and Wald (1984, p. 200). Geroch (1970) originally introduced the notion using "timelike curve" instead of "causal curve" as is now standard. The resulting future domains of dependence differ insofar as the standard domain is a proper subset of the non-standard domain à la Geroch: the latter, but not the former includes the boundary points of $D^{+}(\Sigma)$. Manchak shows that the adoption of Geroch's original definition can be exploited to show that surgery on Misner spacetime as performed in Fig. 4b cannot preclude the emergence of CTCs in all maximal extensions of $D^{+}(\Sigma)$. The removed strips in Fig. $4 \mathrm{~b}$ cut all the way into $H^{+}(\Sigma)$. This is no longer permissible under Geroch's definition because it includes the Cauchy horizon $H^{+}(\Sigma)$ in $D^{+}(\Sigma)$. Manchak shows that smoothly extending this non-standard future domain of dependence of Misner spacetime will always allow just enough of a window for CTCs to slip through below the cut. We reject this alternative conception of $D^{+}(\Sigma)$ because it would allow causal signals that have not emanated from the time slice under consideration to reach points in the future domain of dependence. But this would frustrate our attempts at offering the strongest possible sense in which the CTCs are due to the operation of the potential time machine. As a consequence of our rejection of the alternative conception of $D^{+}(\Sigma)$, hole freeness must be used to obtain the result that Misner spacetime satisfies the Potency Condition, which is not necessary on the alternative conception as shown by Manchak. For the proof to go through, one does not need to require hole freeness of the entire maximal extension of $D^{+}(\Sigma)$, but only of the closure of $D^{+}(\Sigma)$. We maintain that the stronger demand of hole freeness for the entire maximal extension is still preferable since we want to rule out in general artifical cutting strategies attempting to foil the formation of CTCs, as exemplified in Fig. 4b. If CTCs fail to occur, it should be for a better reason than God using his scissors to cut the CTCs. 


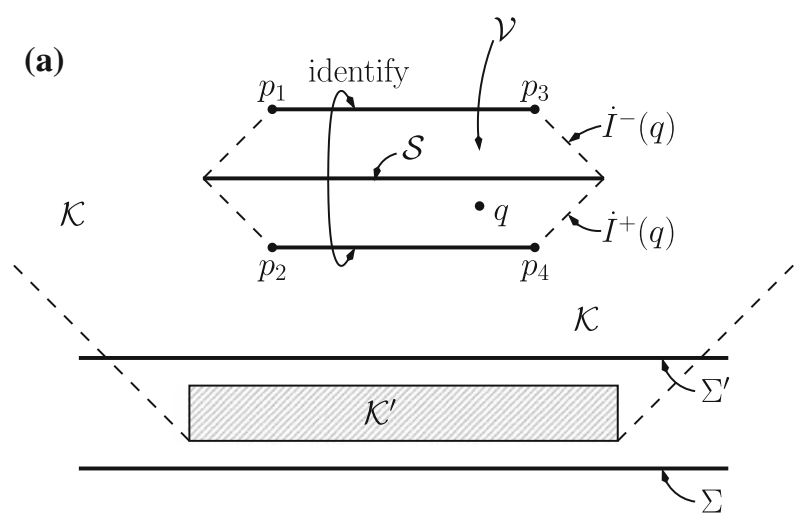

(b)

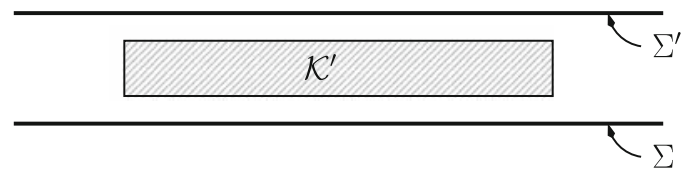

Fig. 5 (a) Deutsch-Politzer spacetime, (b) Minkowski spacetime

Krasnikov (1998) constructs a spacetime which contains a time machine in this sense, which is singularity free, and which satisfies the weak energy condition (the significance of this condition is discussed in the following section). The construction proceeds in two steps. First, construct a Deutsch-Politzer spacetime (see Fig. 5a) by deleting the points $p_{1}-p_{4}$ from Minkowski spacetime and then gluing together the strips as shown. Because of the missing points the spacetime is singular in the sense of geodesic incompleteness. ${ }^{33}$ This is taken care of in the second step. The introduction of an appropriate conformal factor leaves the causal structure the same but makes the spacetime geodesically complete (and, indeed, b-complete ${ }^{34}$ ) without violating the weak energy condition.

The Definition is satisfied if $\mathcal{K}$ is chosen to be the causal future of the shaded rectangle shown in Fig. 5a and $\mathcal{K}^{\prime}$ is chosen to be the shaded region of Minkowski spacetime shown in Fig. 5b. But this reveals the defect of the definition: there is no separation between the operation of the supposed time machine and the CTCs it is supposed to produce. To overcome this defect one could modify the Definition to require that $\mathcal{V} \subset J^{+}(\mathcal{K})$ and that $\mathcal{K}$, like $\mathcal{K}^{\prime}$, has compact closure. The modified definition is satisfied if $\mathcal{K}$ is chosen to be the shaded rectangle in Fig. 5a rather than its causal future. But now another glaring defect emerges: there is no reasonable sense in which this new $\mathcal{K}$ corresponds to the operation of a time machine. Up to "time" $\Sigma$ ', which is after the alleged time machine has operated, the Deutsch-Politzer spacetime and

\footnotetext{
33 A spacetime is geodesically complete just in case every geodesic can be extended to arbitrarily large values of one of its affine parameters.

34 b-completeness is stronger than geodesic completeness. For a definition of b-completeness see Hawking and Ellis (1973, p. 259).
} 
the corresponding portion of Minkowski spacetime (Fig. 5b) are isometric. So there is no reasonable sense in which the CTCs in Deutsch-Politzer spacetime are "due to" the conditions in the time machine region $\mathcal{K}$ - or for that matter to the entirety of the conditions prior to $\Sigma^{\prime}$, which may be chosen to lie as close to the chronology violating region $\mathcal{V}$ as desired. For no interesting form of the Potency Condition is fulfilled in this case since there are extensions beyond $\Sigma^{\prime}$-e.g. to Minkowski spacetime-which do not contain CTCs but which satisfy every condition that could be reasonably demanded of a suitable extension.

If the Potency Condition is accepted as the key to seeing a time machine at work, then one could seek to motivate Hawking's condition of compactly generated future Cauchy horizon by proving a form of the conjecture that, in some broad and interesting class of spacetimes, when $H^{+}(\Sigma)$ is compactly generated, the Potency Condition is realized for $D^{+}(\Sigma)$. The seeming plausibility of such a conjecture follows from the fact that strong causality is violated in a neighborhood of $H^{+}(\Sigma)$ if it is compactly generated. ${ }^{35}$ (Strong causality is violated at a point $p$ if, intuitively, there are almost closed causal curves near $p$; more precisely, there is a neighborhood of $p$ such that every subneighborhood has the property that some causal curve intersects it more than once.) This violation can be taken as an indication that the seeds of acausality have been planted in $D^{+}(\Sigma)$ and are ready to bloom in the form of CTCs in any non-artificially constructed maximal extension. But to the extent that the Potency Condition can be satisfied in the absence of a compactly generated Cauchy horizon, Hawking's condition cannot be counted as a necessary condition for the operation of a time machine.

At first blush the suggested approach to identifying the operation of a time machine by means of the Potency Condition is threatened by a result of Krasnikov (2002) showing that every time oriented spacetime $\mathcal{M}, g_{a b}$ without CTCs has as an extension a maximal time oriented spacetime such that any CTC in the extension lies to the chronological past of the image of $\mathcal{M}$ in the extension. Furthermore, the construction allows local conditions on the metric, such as Einstein's field equations and energy conditions, to be carried over to the maximal extension. But at second glance, it is not at all obvious that Krasnikov's construction guarantees that his maximal extension will satisfy our preferred form of the Potency Condition that includes a hole freeness restriction on extensions. And the result of Manchak (2007) mentioned above indicates that the Potency Condition in our preferred form is not empty.

In the next section we start to include among the requirements asked of suitable extensions $H^{+}(\Sigma)$ various constraints based on dynamics. But before turning to this task we want to acknowledge an alternative approach. Our method of specifying what it means for a would-be time machine to be "responsible for" the formation of CTCs appeals to features of the causal structure of the spacetimes of classical GTR. Alternatively, one could try to cash out "responsible for" using one or another of the analyses of causation available in the philosophical literature. The three leading analyses are (i) interventionist, (ii) counterfactual, and (iii) probabilistic. The first seems

\footnotetext{
35 This follows from the result that the generators of $H^{+}(\Sigma)$ for a partial Cauchy surface $\Sigma$ are past endless. If $H^{+}(\Sigma)$ is compactly generated, we thus have a past endless causal curve totally imprisoned in a compact set, and thus by Prop. 6.4 .7 of Hawking and Ellis (1973, p. 195) strong causality fails in this set.
} 
inappropriate when the system at issue is the entire cosmos. The second and third require an apparatus that is not provided by GTR itself - in the case of (ii) a nearness relation on the possible worlds (here, solutions to Einstein's field equations) and in the case of (iii) a probability measure over these worlds. Providing such an apparatus and discussing its implications for time machines is a worthy enterprise. But it is not one that we will pursue here since it quickly loses contact with the physics literature and threatens to mire the discussion of time machines in the endless controversies that swirl around the cause-effect relation.

\section{Chronology protection and no-go results for time machines in classical GTR}

The absence of a satisfactory analysis of "time machine" in the sizable and ever growing physics literature on this topic is less scandalous than it might seem at first impression, at least for that portion of the literature that aims at no-go results; for the authors of these results don't have to offer an analysis but only necessary conditions for the operation of a time machine. Our complaint about this literature does not derive from the lack of a general analysis but from the fact that a scrutiny of the proffered necessary conditions often reveals a dubious quality that undermines the force of the no-go result.

The program of producing no-go results for time machines can be seen as part of the more ambitious program of producing chronology protection theorems that take the form: "If no CTCs are present to begin with, then CTCs cannot emerge without violating conditions ," where the conditions that go into the blank are to be justified on the grounds that they must be satisfied in any physically reasonable model of GTR. (Hawking's original version of the chronology protection conjecture stated that: "The laws of physics do not allow the appearance of closed timelike curves" (1992a, p. 603).) Establishing that CTCs cannot be manufactured by time machines would go part way-but only part way - towards establishing chronology protection. In the other direction, a strong chronology protection theorem would put the time machine industry out of business without having to settle on necessary and sufficient conditions for a time machine. In turn, the program of establishing chronology protection can be seen as part of the still more ambitious program of establishing a strong version of cosmic censorship, which militates against the formation of "naked singularities" in the form of any pathology that would undermine global hyperbolicity (see Penrose (1998)).

In preparation for the discussion of chronology protection results, we will comment briefly on the role of energy conditions. Einstein's field equations set the Einstein tensor $G_{a b}$, which is computed from the metric $g_{a b}$ and its derivatives, proportional to the stress-energy tensor $T_{a b}$, which describes the distribution of matter-energy. Save for the vacuum case $\left(T_{a b} \equiv 0\right)$ the imposition of Einstein's field equations has no bite unless restrictions are put on possible values for $T_{a b}$; for given any smooth metric $g_{a b}$, Einstein's equations are satisfied for the $T_{a b}$ simply set equal to $G_{a b} / 8 \pi$ (in geometrical units, where $G=c=1$ ). The field equations do have considerable bite when conjoined with the requirement that $T_{a b}$ must arise from known matter fields. But adopting this requirement would mean that chronology protection theorems could not have the ambition of showing that chronology is protected for as yet unknown but 
physically reasonable fields. For this reason chronology protection theorems adopt instead one or another condition on $T_{a b}$ thought to hold for most, if not all, physically reasonable fields.

The most widely cited of these conditions is called the weak energy condition (WEC), which says intuitively that there are no negative energy densities. The technical statement is that $T_{a b} V^{a} V^{b} \geq 0$ for any timelike $V^{a}$. For a perfect fluid with density $\mu$ and pressure $p$ this is equivalent to requiring $\mu \geq 0$ and $\mu+p \geq 0$. The strong energy condition (SEC) which, despite what its name suggests, does not entail the WEC, requires that $T_{a b} V^{a} V^{b} \geq \frac{1}{2} \operatorname{Tr}\left(T_{a b}\right)$ for every unit timelike $V^{a}$. The dominant energy condition (DEC) says intuitively that the energy-momentum flow as measured by any observer is non-spacelike. Technically the condition is that for any future-directed timelike $V^{a},-T_{a b} V^{a}$ is a future-directed null or timelike vector. ${ }^{36}$

Satisfaction of these energy conditions is sometimes taken as the defining feature of physically reasonable matter. But these conditions are not sacrosanct; they can fail to hold for quantum fields, and recently physicists have contemplated matter sources violating one or more of the energy conditions. To take a recent example, in standard Friedmann-Robertson-Walker cosmological models the expansion of the universe decelerates if the matter sources satisfy the WEC and the SEC. ${ }^{37}$ To capture the observed accelerated expansion in these models, there must be a form of matter, "dark energy," violating the SEC. ${ }^{38}$ One candidate for the dark energy called "phantom matter" violates all three of the above energy conditions. It has been suggested that such matter could be used to produce traversable wormholes and thence CTCs (see Lobo (2005)); but because the violation of the DEC by such matter opens to door to acausal propagation, it is not clear how seriously the suggestion should be taken.

Early no-go results for time machines were proved by Tipler (1976, 1977). His results showed that the evolution of CTCs from regular initial data on an asymptotically flat partial Cauchy surface would, under certain conditions, lead to a violation of the conjunction of the Einstein field equations and the WEC, or else would cause spacetime singularities to form. Thus, if to "manufacture" CTCs means "construct using only ordinary matter everywhere" and "ordinary matter" satisfies the WEC and does not reach arbitrarily great densities, then the theorems do count as no-go results for time machines. Or at least they would if the sense of "singularity" used in the proof of the theorems-namely, geodesic completeness-entailed the existence of arbitrarily large matter densities; but this entailment is not universal. In particular it fails to hold for typical time machine models, in which the presence of an incomplete null geodesic does not signal divergent matter densities. ${ }^{39}$ A second and more important limitation (noted by Ori 1993) is that Tipler's theorems do not contain any information about

\footnotetext{
36 The minus sign is a result of our choice of signature $(+++-)$ for the spacetime metric.

37 More precisely, $\ddot{a}<0$ if the WEC and SEC are satisfied, where $a(t)$ is the scale factor in the FRW models.

38 For a review of the dark energy problem, see S. Carroll (2004).

39 For example, Hawking (1992a) discusses a time machine model including a "fountain" of the compactly generated Cauchy horizon, an incomplete null geodesic, which is not associated with divergent energy densities.
} 
when the singularities form; if they do not form prior to the emergence of CTCs, then even if they do entail unbounded matter densities they cannot serve as an explanation of why CTCs cannot be manufactured from ordinary matter.

The next significant chronology protection result was due to Stephen Hawking. If $\Sigma$ is a partial Cauchy surface and $H^{+}(\Sigma)$ is compact, then $\Sigma$ must be compact (Hawking 1992a; Chrusciel and Isenberg 1993). This is a purely geometrical result. ${ }^{40}$ Thus, whether or not Einstein's field equations or energy conditions hold, if $\Sigma$ is non-compact (i.e. the universe is spatially open), then $H^{+}(\Sigma)$ is non-compact. But can $H^{+}(\Sigma)$ nevertheless be compactly generated when $\Sigma$ is non-compact? Hawking (1992a) showed that the answer is no under plausible assumptions. Specifically, he proved that if $\Sigma$ is non-compact and if Einstein's field equations and the WEC are satisfied, then $H^{+}(\Sigma)$ cannot be compactly generated.

How effective is Hawking's result in protecting chronology? The first comment is that the WEC is not absolutely sacred even in classical GTR. Vollick (1997) has shown how the WEC can be violated using a charged dust interacting with a scalar field. Although the dust and the scalar field separately have positive energy densities, the interaction part of the stress-energy tensor can have negative energy densities. Using this fact, Vollick showed that it is possible to build a sphere of charged dust with negative energy density throughout the interior. ${ }^{41}$

But apart from doubts about the WEC, the crucial issue is whether the condition of compactly generated Cauchy horizons can be justified as a necessary condition for the operation of a time machine since it certainly cannot be justified as a condition that any physically reasonable model of GTR must fulfill. By our lights the justification would have to proceed by showing that when $H^{+}(\Sigma)$ is not compactly generated, a strong and interesting form of the Potency Condition necessarily fails. This seems implausible, for there is no evident reason why, in the generic case of a four-dimensional inhomogeneous spacetime, it cannot happen that the behavior of the metric in one region of $D^{+}(\Sigma)$ implies that all smooth hole free extensions that satisfy Einstein's field equations and energy conditions contain CTCs, while overall the behavior of the metric implies that $\mathrm{H}^{+}(\Sigma)$ is not compactly generated in some or all such extensions (because, for example, curvature singularities develop in other regions). ${ }^{42}$ Of course, this objection falls flat if our preferred version of the Potency Condition cannot be satisfied for the class of models being considered. But in that case the issue of whether Hawking's condition of compactly generated Cauchy horizons is necessary for the

\footnotetext{
40 Assuming that the spacetime is time orientable, there always exists a continuous non-vanishing timelike vector field. Using the integral curves of this vector field, map the points of $H^{+}(\Sigma)$ into the interior $D^{+}(\Sigma)$. This mapping is one-to-one and onto. Thus, if $H^{+}(\Sigma)$ is compact, so is the resulting surface. In addition, the resulting surface is spacelike and diffeomorphic to $\Sigma$.

41 Vollick shows that for a sphere of charged particles coupled to a scalar field, $T_{44}<0$ in the interior of a sphere provided that $\left(\frac{\alpha}{m}\right)^{2} \mu_{o} R^{2}>2$, where $\alpha$ is the coupling constant between matter and the scalar field, $\mu_{o}$ is the rest mass density, and $R$ is the radius of the sphere. Although this violation of the weak energy condition is achieved without recourse to zero-point energies and other subtleties of quantum field theory, the scalar field must be very strongly coupled to matter over large distances. We take the existence of such a scalar field to be physically implausible.

42 Doubts about Hawking's condition of compactly generated Cauchy horizon as a sine qua non for time machines have also been expressed by Ori (1993) and Krasnikov (1999).
} 
operation of a time machine is moot since it is unclear how to characterize a time machine for the class of models under consideration.

Hawking's theorem does not apply when the partial Cauchy surface $\Sigma$ is compact, for otherwise Taub-NUT spacetime and Misner spacetime would be counterexamples. To attempt to fill the gap, one can bring to bear Theorem 3 of Tipler (1977). The proof of this theorem can be adapted to show that if the WEC and Einstein's field equations hold and if the generic condition holds somewhere on $H^{+}(\Sigma)(\neq \varnothing)$, then $H^{+}(\Sigma)$ is not compactly generated. Taub-NUT spacetime and Misner spacetime are not counterexamples because they violate the generic condition which requires that $V^{a} V^{b} V_{[c} R_{d] a b[e} V_{f]}=0$, where $R_{a b c d}$ is the Riemann tensor and $V^{a}$ is the tangent to a timelike or null geodesic. The generic condition entails that all geodesics feel a tidal force. The absence of such a force on all of $H^{+}(\Sigma)$ would signal that the spacetime is of a rather "special" character. ${ }^{43}$ But then no one thought that spacetimes implementing time machines would be generic. One expects more from a no-go result than a statement of the form: a time machine can operate only under such-and-such special conditions.

Hawking (1992a) attempted to fill the gap in another way. When $\Sigma$ is compact, $H^{+}(\Sigma)$ can be compactly generated. But if it is, both the convergence and shear of the null geodesic generators of $H^{+}(\Sigma)$ must vanish if the WEC holds. "This would mean," Hawking wrote, "that no matter or information, and in particular no observers, could cross the Cauchy horizon into the region of closed timelike curves" (p. 606). But this implication doesn't constitute an anti-time machine result per se; it says rather that the operator of the time machine cannot take advantage of his handiwork.

Two further results deserve brief mention. Maeda et al. (1998) prove that there is no maximal spacetime containing a non-empty chronology violating set $\mathcal{V}$ if a number of conditions are satisfied. The theorem is self-consciously styled as a no-go result for time machines, but as such it is aimed mainly at finitely vicious spacetimes (where $\mathcal{V}$ is contained in a compact set) that arise from the sorts of identifications made in the construction of Deutsch-Politzer spacetime. We argued in the preceding section that Deutsch-Politzer spacetime is not a plausible candidate for a time machine spacetime, so this theorem does not recommend itself as a general chronology protection theorem. Another recent no-go result operates against the possibility of producing CTCs by means of traversable wormholes: Hochberg and Visser $(1997,1999)$ establish the violation of energy conditions at or near the throats of traversable wormholes.

In sum, while the various theorems reviewed above that have been advertised as no-go results for time machines or as chronology protection theorems for classical GTR do indicate that there are problematic aspects to implementing a time machine in this context, they do not justify the gloss that the laws of classical GTR forbid the operation of a time machine.

\footnotetext{
43 There are other results which further suggest that a time machine spacetime must be "special." For example, Isenberg and Moncrief (1985) considered analytic vacuum and electrovacuum solutions to EFE which contain a compact null hypersurface ruled by closed null geodesics. They showed that such spacetimes must be special in the sense that they possess a Killing symmetry.
} 


\section{No-go results using semi-classical quantum gravity}

The introduction of quantum considerations cuts in two directions with respect to proving chronology protection results and/or no-go results for time machines: in one direction the quantum would appear to undermine the classical no-go results since quantum fields can, for example, violate the WEC assumed in many of these results; ${ }^{44}$ but in the other direction the quantum introduces new mechanisms that may prevent the formation of CTCs. In this section we study one such mechanism which arises in the context of semi-classical quantum gravity (SCQG).

The ambitions of SCQG do not extend to quantizing the metric; rather the aim is to estimate how quantum fields affect the metric by computing the quantum expectation value $\left\langle\xi\left|T_{a b}\right| \xi\right\rangle$ of the (renormalized) stress-energy tensor $T_{a b}$ that arises from the quantum fields, and then by inserting this expression into Einstein's field equations in place of the classical stress-energy tensor in order to calculate the "backreaction" of the fields on the metric. Work by Frolov (1991) and Kim and Thorne (1991) suggested that for physically interesting states $|\xi\rangle$ of linear quantum fields, $\left\langle\xi\left|T_{a b}\right| \xi\right\rangle$ diverges as the Cauchy horizon $\mathrm{H}^{+}(\Sigma)$ is approached in classical general relativistic spacetimes where CTCs occur to the future of $H^{+}(\Sigma)$. Hawking (1992a) argued that when the backreaction has an attractive gravitational effect, the divergence behavior would cause the formation of singularities along $H^{+}(\Sigma)$, cutting off future development of the spacetime, whereas when the backreaction has a repulsive gravitational effect "the spacetime will resist being warped so that closed timelike curves appear" (p. 610); either way, the mechanism of SCQG acts to prevent the formation of CTCs. Hawking's argument has not been made rigorous; indeed, it cannot be made rigorous in our present state of knowledge since, presumably, the details will depend on the presently non-existent quantum theory of gravity.

Basing a no-go theorem on the divergence of $\left\langle\xi\left|T_{a b}\right| \xi\right\rangle$ faces a more immediate obstacle: in some cases there is no divergence! Krasnikov (1996) and Sushkov (1997) studied some toy models with CTCs and a partial Cauchy surface $\Sigma$ and exhibited states $|\xi\rangle$ for which $\left\langle\xi\left|T_{a b}\right| \xi\right\rangle$ remains bounded as $H^{+}(\Sigma)$ is approached. ${ }^{45}$ The "Sushkov state" is not entirely well behaved since the expectation values of other field quantities diverge as the Cauchy horizon is approached. If anything, these results show that one can circumvent the heuristic arguments for the divergence of $\left\langle\xi\left|T_{a b}\right| \xi\right\rangle$ by cleverly choosing the field and/or the spacetime. ${ }^{46} \mathrm{~A}$ general problem with these results is that

\footnotetext{
44 However, Ford and Roman (1996) argue that "quantum inequalities" place limitations on the violation of the weak energy condition and that in turn these limitations constrain the geometry of traversable wormholes that might allow CTCs. For a review of more recent results along these lines see Fewster (2005).

45 Sushkov (1997) proved that for an automorphic quantum field (characterized as a complex scalar field $\varphi$ in an external electromagnetic field) in four-dimensional Misner space, carefully setting the automorphic parameter yields a field such that $\left\langle\xi\left|T_{a b}\right| \xi\right\rangle$ is zero everywhere on the initially globally hyperbolic region $D^{+}(\Sigma)$. An automorphic field at a point $x$ satisfies the following condition: $\varphi(L x)=\exp (2 \pi i \alpha) \varphi(x)$, where $L$ is an element of the spacetime isometry group and $0 \leq \alpha \leq \frac{1}{2}$ is the automorphic parameter.

46 Hiscock (2000) has rejected these and other purported counterexamples to Hawking's chronology protection conjecture on the grounds that they either rely on special properties of the quantum fields or else they depend on "fine tuning" the metric parameters. In a similar vein, purported counterexamples to Roger Penrose's cosmic censorship hypothesis have been rejected on the grounds that they are highly non-generic.
} 
even if there is a well-defined limit of $\left\langle\xi\left|T_{a b}\right| \xi\right\rangle$ as the horizon is approached, the value of $\left\langle\xi\left|T_{a b}\right| \xi\right\rangle$ on the horizon may still be singular. In the Sushkov state the renormalized stress energy tensor vanishes on the initially globally hyperbolic region, but it vanishes on the Cauchy horizon as well only if continuity holds (see Cramer and Kay 1996). A result due to Kay et al. (1997) (hereafter, KRW) shows that continuity necessarily fails: $\left\langle\xi\left|T_{a b}\right| \xi\right\rangle$ cannot be non-singular on all of $H^{+}(\Sigma)$ when $H^{+}(\Sigma)$ is compactly generated. ${ }^{47}$ In any case, physicists gave up on the idea that the "blow up" of $\left\langle\xi\left|T_{a b}\right| \xi\right\rangle$ at the chronology horizon provides a mechanism for chronology protection.

Rather than focusing on the behavior of field properties as $H^{+}(\Sigma)$ is approached, KRW prove that the formation of CTCs undermines the applicability of SCQG. Understanding their result requires a brief review of the "point-splitting prescription" for evaluating $\left\langle\xi\left|T_{a b}\right| \xi\right\rangle$ (see Wald 1994, Ch. 4 and KRW). The stress energy tensor for a scalar field $\varphi$ typically includes $\varphi^{2}$ terms. ${ }^{48}$ There is no natural way to define a product of distributions at a single point. As the name suggests, in point-splitting the product is treated as the coincidence limit of a bidistribution defined over two points, e.g. $\left\langle\xi\left|\varphi^{2}(x)\right| \xi\right\rangle:=\lim _{x \rightarrow x^{\prime}}\left[\left\langle\xi\left|\varphi(x) \varphi\left(x^{\prime}\right)\right| \xi\right\rangle-H\left(x, x^{\prime}\right)\right] . H\left(x, x^{\prime}\right)$ corresponds to "vacuum stress-energy" and it is subtracted to get rid of possible divergences. The quantity in square brackets is a smooth, well-defined function of $x$ and $x^{\prime}$ only if the two terms have the same short range singularity behavior. In Minkowski spacetime $H\left(x, x^{\prime}\right)$ for the preferred vacuum state is singular if and only if the points $\left(x, x^{\prime}\right)$ are null related. So the procedure yields a well-defined quantity $\left\langle\xi\left|\varphi^{2}(x)\right| \xi\right\rangle$ if $\left\langle\xi\left|\varphi(x) \varphi\left(x^{\prime}\right)\right| \xi\right\rangle$ is required to have the same singular behavior as $H\left(x, x^{\prime}\right)$.

Generalizing this approach to curved spacetimes leads to the requirement that physically admissible states satisfy what is called the Hadamard condition. Curved spacetimes lack a preferred global vacuum state, so to apply the point-splitting prescription one needs to determine the properties of $H\left(x, x^{\prime}\right)$. As Wald (1994) shows, specifying four desirable properties for $\left\langle\xi\left|T_{a b}\right| \xi\right\rangle$ leads to constraints on $H\left(x, x^{\prime}\right)$; roughly, the upshot is that $H\left(x, x^{\prime}\right)$ is singular for null-related points but well-defined for other points (it is "locally weakly Hadamard"). The Hadamard condition limits physically admissible states to those whose two-point functions $\left\langle\xi\left|\varphi(x) \varphi\left(x^{\prime}\right)\right| \xi\right\rangle$ have the same singularity structure as $H\left(x, x^{\prime}\right)$.

KRW's result shows that in a neighborhood of a base point of a compactly generated Cauchy horizon there is no way to consistently define a Hadamard state. The set of base points $\mathcal{B}$ is defined as the set of points $p$ which are past terminal accumulation points for some null geodesic generator $\gamma$ of $H^{+}(\Sigma)$-intuitively, $\gamma$ continually re-enters

\footnotetext{
47 Cramer and Kay (1996) generalize the KRW result slightly to cover Sushkov's example; $H^{+}(\Sigma)$ is not compactly generated in four-dimensional Misner spacetime considered by Sushkov, but the KRW result applies to any manifold which is the product of an $n$-dimensional spacetime with a compactly generated Cauchy horizon and a $(4-n)$-dimensional Riemannian manifold.

${ }^{48}$ For a Klein-Gordon field, for example, $T_{a b}=\nabla_{a} \varphi \nabla_{b} \varphi-\frac{1}{2} g_{a b}\left(\nabla_{c} \nabla^{c} \varphi+m^{2} \varphi^{2}\right)$.
} 
any given neighborhood of the point $p .{ }^{49}$ (In the case of the Misner spacetime of the preceding section, every point of $H^{+}(\Sigma)$ is a base point.) KRW prove that

Lemma 1 (a) When $H^{+}(\Sigma)$ is compactly generated, $\mathcal{B} \neq \emptyset$. (b) For any globally hyperbolic neighborhood $\mathcal{U}$ of a base point, there are points $y, z \in \mathcal{U} \cap D^{+}(\Sigma)$ such that $y$ and $z$ are connected by a null geodesic in $\mathcal{M}, g_{a b}$ but cannot be connected by a null curve lying entirely in $\mathcal{U}$.

(The reader should verify property (b) for the case of Misner spacetime.) Because of the clash between the local and global senses of null-related points and because the singularity structure of a Hadamard state depends on which points are null related, the point splitting procedure fails to yield a well-defined value for $\left\langle\xi\left|T_{a b}\right| \xi\right\rangle$ at a base point. $^{50}$

It is expected that analogous results hold for cases where $H^{+}(\Sigma)$ is not compactly generated but serves as a chronology horizon in the sense that it separates the initially globally hyperbolic region $D^{+}(\Sigma)$ from a region where there are CTCs (see Cramer and Kay 1996; KRW 1997). The collection of such results promises to serve as a basis for a general chronology protection theorem to the effect that the laws of physics prevent the formation of CTCs in a spacetime initially free of causal anomalies, whether or not the formation can be attributed to a time machine. But exactly how is this promise to be realized? The original idea was that because the expectation value of the stress-energy tensor "blows up" as the chronology horizon is approached, backreaction effects shut down the incipient formation of CTCs. That idea, to repeat, has been shot down by a number of counterexamples. The new approach, while much more rigorous, is explanatorily less satisfying in that it doesn't provide a mechanism for preventing the formation of CTCs; rather it proceeds by showing that, under suchand-such conditions, the formation of CTCs is inconsistent with the applicability of SCQG, which requires that expectation value of the stress-energy tensor be welldefined on $H^{+}(\Sigma)$. As is often the case with reductio arguments, this reductio leaves the beholder searching for the explanatory force.

Visser $(1997,2003)$ has argued that the situation is even worse because the chronology horizon lies beyond the "reliability horizon," which marks the limit beyond which SCQG cannot be reliably applied. The "unreliable region" $\Omega$ consists of points connected to themselves by spacelike geodesics shorter than the Planck length (ca. $10^{-45} \mathrm{~m}$ ), and the reliability horizon is the boundary of $J^{+}(\Omega)$. The existence of these closed Planck scale loops spell disaster for SCQG because the mode sum for a quantum field defined over a region with such periodic spatial identifications will include momentum terms on the order of the Planck energy. These energies and

\footnotetext{
49 More precisely, a past terminal accumulation point of a curve $\gamma: I \rightarrow \mathcal{M}, I \subset \mathbb{R}$, is defined as a point $p \in \mathcal{M}$ such that for every open neighborhood $\mathcal{N}(p)$ and every $s \in I, \exists\left(s^{\prime} \in I: s^{\prime}>s \& \gamma\left(s^{\prime}\right) \in \mathcal{N}(p)\right]$, where $\gamma$ is parametrized so as to be past directed. Since the null geodesic generators of $H^{+}(\Sigma)$ are past inextendible, for every base point there is a null curve which intersects any given neighborhood of that point more than once. Thus, strong causality fails at each base point.

50 The no-go result also relies on the propagation of singularities theorem which says, very roughly, that singularities in a bi-solution to the Klein-Gordon field equation for nearby points on a null geodesic are propagated along the entire geodesic.
} 
associated metric fluctuations are great enough that we should not trust SCQG to give an accurate rendering of backreaction effects.

The summing up of this section echoes that of the previous section: while results from SCQG reveal problematic features of attempts to implement time machines or, more generally, violations of chronology protection, they do not add up to impossibility proofs.

\section{No-go results from quantum field theory on curved spacetime}

If SCQG is a kind of first order approximation to full quantum gravity, then quantum field theory (QFT) on curved spacetime is a 0th order approximation: it has no ambition to compute backreaction effects; rather, a spacetime of classical GTR is treated as a fixed background on which one attempts to do QFT. There is a general recipe for cooking up a natural algebra of observables $\mathfrak{A}\left(\mathcal{M}, g_{a b}\right)$ for a linear quantum field $\varphi$ defined over a globally hyperbolic spacetime $\mathcal{M}, g_{a b}$ (see Wald 1994). The result of this recipe is a $C^{*}$-algebra generated by the elements representing "smeared" quantum fields $\varphi(f)$, where $f$ belongs to a suitable "test function" space. ${ }^{51}$ This procedure for constructing a QFT on curved spacetime breaks down for non-globally hyperbolic spacetimes, and one can wonder whether it is even possible to construct a decent QFT when the spacetime is so causally ill-behaved as to contain CTCs and time machines. This opens the door for another approach to proving chronology protection theorems; namely, state and defend as minimally necessary a condition that a spacetime must satisfy in order to admit a decent QFT; then show that if a spacetime is initially free of CTCs, it can develop CTCs only at the price of violating said minimally necessary condition.

Kay (1992) has argued that a requirement he dubs $F$-quantum compatibility is just such a minimally necessary condition. To explain it, note first that even if a spacetime $\mathcal{M}, g_{a b}$ fails to be globally hyperbolic, it is still possible to choose for any $p \in \mathcal{M}$ a globally hyperbolic neighborhood $\mathcal{U} \subset \mathcal{M}$, i.e. $\mathcal{U}, g_{a b} \mid \mathcal{U}$ considered as a spacetime in its own right is globally hyperbolic. One can then proceed per usual to construct the intrinsic algebra $\mathfrak{A}\left(\mathcal{U}, g_{a b} \mid \mathcal{U}\right)$ for this spacetime. But another possible choice of an algebra associated with $\mathcal{U}$ is the algebra $\mathfrak{A}\left(\mathcal{M}, g_{a b} ; \mathcal{U}\right)$ induced on $\mathcal{U}$ by the global algebra $\mathfrak{A}\left(\mathcal{M}, g_{a b}\right){ }^{52} \mathcal{M}, g_{a b}$ is said to satisfy $F$-locality with respect to a given $\mathfrak{A}\left(\mathcal{M}, g_{a b}\right)$ just in case for every $p \in \mathcal{M}$ there is a globally hyperbolic neighborhood $\mathcal{U}$ such that the algebras $\mathfrak{A}\left(\mathcal{U}, g_{a b} \mid \mathcal{U}\right)$ and $\mathfrak{A}\left(\mathcal{M}, g_{a b} ; \mathcal{U}\right)$ are isomorphic. And $\mathcal{M}, g_{a b}$ is said to be $F$-quantum compatible just in case it admits a global algebra $\mathfrak{A}\left(\mathcal{M}, g_{a b}\right)$ with respect to which it is F-local.

In order to relate F-locality to chronology protection we need to be more specific about how the algebras are constructed. Here we restrict attention to a scalar quantum field $\varphi$ satisfying the Klein-Gordon equation $\left(\square_{g}-m^{2}\right) \varphi=0$, where $\square_{g}$ is the

\footnotetext{
${ }^{51}$ E.g. $C_{0}^{\infty}(\mathcal{M})$ (i.e. smooth functions with compact support on $\mathcal{M}$ ). The smeared field is defined by $\varphi(f):=\int_{\mathcal{M}} \varphi(x) f(x) \eta$, where $\eta$ is the volume element for the spacetime.

$52 \mathfrak{A}\left(\mathcal{M}, g_{a b} ; \mathcal{U}\right)$ is the $C^{*}$-completion of the set of all $\mathfrak{A}\left(\mathcal{M}, g_{a b}\right)(\mathcal{O})$ for which the closure of the open set $\mathcal{O}$ is contained in $\mathcal{U}$, and $\mathfrak{A}\left(\mathcal{M}, g_{a b}\right)(\mathcal{O})$ is the subalgebra of $\mathfrak{A}\left(\mathcal{M}, g_{a b}\right)$ generated by the smeared fields on $\mathcal{M}$ with $f \in C_{0}^{\infty}(\mathcal{O})$.
} 
Laplace-Beltrami operator with respect to the metric $g_{a b}$ and $m \geq 0$ is the mass of the field. When $\mathcal{M}, g_{a b}$ is globally hyperbolic, $\mathfrak{A}\left(\mathcal{M}, g_{a b}\right)$ is taken to be the $C^{*}$-algebra of smeared quantum fields satisfying the following conditions (KRW 1997):

1. $\varphi(f)=\varphi(f)^{*}$

2. $\varphi\left(\lambda_{1} f_{1}+\lambda_{2} f_{2}\right)=\lambda_{1} \varphi\left(f_{1}\right)+\lambda_{2} \varphi\left(f_{2}\right)$

3. $\varphi\left(\left(\square_{g}-m^{2}\right) f\right)=0$

4. $\left[\varphi\left(f_{1}\right), \varphi\left(f_{2}\right)\right]=i \Delta\left(f_{1}, f_{2}\right)$

where $\Delta$ is the advanced-minus-retarded fundamental solution to the Klein-Gordon equation. The existence and uniqueness of $\Delta$ is guaranteed for globally hyperbolic spacetimes. This guarantee fails when the spacetime is not globally hyperbolic. Nevertheless one can construct for any globally hyperbolic neighborhood $\mathcal{U}$ the $C^{*}$-algebra of smeared fields such that condition 4 holds for $f_{1}, f_{2} \in C_{0}^{\infty}(\mathcal{U})$ with $\Delta$ replaced by $\left.\Delta\right|_{\mathcal{U}}$, the advanced-minus-retarded fundamental solution for $\mathcal{U}, g_{a b} \mid \mathcal{U}$.

Now consider a spacetime where $H^{+}(\Sigma)$ is compactly generated. We know from the Lemma of the previous section that the set $\mathcal{B} \subset H^{+}(\Sigma)$ of base points is non-empty. Let $\mathcal{U}$ be any globally hyperbolic neighborhood of a base point. Then the restrictions of $\mathfrak{A}\left(\mathcal{U}, g_{a b} \mid \mathcal{U}\right)$ and of $\mathfrak{A}\left(D^{+}(\Sigma),\left.g_{a b}\right|_{D^{+}(\Sigma)}\right)$ to $\mathcal{U} \cap D^{+}(\Sigma)$ cannot coincide, as follows from the Lemma. It is a basic property of $\Delta$ that it vanishes for spacelike related points, whereas it is singular for null related points. Thus, $\left.\Delta\right|_{\mathcal{U}}$ and $\left.\Delta\right|_{D^{+}(\Sigma)}$ cannot coincide when $\mathcal{U}$ is a globally hyperbolic neighborhood of a base point. This shows that the usual field algebra defined above for initially globally hyperbolic region $D^{+}(\Sigma)$ cannot be extended in any way so as to satisfy F-locality on a compactly generated $H^{+}(\Sigma)$. Under some extra mild technical assumptions KRW (1997) show additionally that there is no field algebra at all that satisfies F-locality on $H^{+}(\Sigma)$ when it is compactly generated.

In assessing the significance of these results for chronology protection, it is worth noting that F-locality is not incompatible with time travel per se: there are spacetimes that contain CTCs but are F-quantum compatible- the spacetime of Fig. 1 being just such a case (see Kay 1992 and Fewster and Higuchi 1996). But F-locality is incompatible with the development of CTCs by means of a process that produces compactly generated $H^{+}(\Sigma)$ or, more generally, a $H^{+}(\Sigma)$ containing base points with the property (b) of the Lemma. This is a chronology protection result, albeit of limited scope. If Hawking's condition of a compactly generated $H^{+}(\Sigma)$ were a necessary condition for the operation of a time machine then the demonstrated incompatibility would be a completely general no-go result for time machines in QFT on classical general relativistic spacetimes. But as we have seen, there is reason to doubt that Hawking's ansatz plays this role.

Apart from issues of scope, the main worry about the effectiveness of the demonstrated incompatibility as a chronology protection theorem revolves around the status of F-locality. Most directly, one can worry whether F-locality constitutes a sine qua non for QFT. Krasnikov (1999) has argued that a modified F-locality condition is perfectly compatible with the existence of a compactly generated Cauchy horizon. If his argument is correct, then the constraint on spacetime structure illustrated by the KRW results merely indicate that the F-locality condition includes what Krasnikov calls an "arbitrary requirement" responsible for the non quantum 
compatibility of spacetimes with compactly generated Cauchy horizons. As it stands, however, Krasnikov's modified F-locality condition is not an adequate replacement for F-locality since it focuses on defining a field algebra over the union of globally hyperbolic subsets of a manifold rather than over the entire manifold. ${ }^{53}$ This leaves out precisely those parts of the manifold where F-locality is a substantial requirement! Friedman (1997) has also suggested a weaker version of a locality principle: instead of requiring that there is a global algebra which satisfies the F-locality condition, he considers a set of local $C^{*}$-algebras with suitable overlap conditions. However, Friedman's considerations are limited to strongly causal spacetimes, and it is not clear that extending his approach to spacetimes with Cauchy horizons will lead to a condition weaker than F-locality. Although this is a subject for further research, as of yet there is no suitable replacement for F-locality which avoids the KRW result.

The results on the incompatibility of F-locality and the development of CTCs does not furnish a mechanism that explains how Nature prevents the formation of CTCs. Presumably that mechanism will have to come from the full theory of quantum gravity of which QFT on curved spacetime is only a 0th order approximation. For sake of argument assume that the sought after quantum theory of gravity has a semi-classical limit in which QFT on curved spacetime emerges as the interaction between the quantized matter fields and the metric is turned off. Why should one think that this limit will always obey F-locality? Kay writes: "One also expects that, if full quantum gravity satisfies a set of local laws, then some remnant of this locality would survive at the semiclassical level and it seems worthwhile to anticipate what this might be" (1992, 171-172). In the absence of even a dim view of the sought after theory of quantum gravity it is not clear why the antecedent should be realized, or why, if it is realized, the semi-classical limit of quantum gravity will conform to F-locality. Still, there is an interesting implication to be drawn from trying to suppose that F-locality fails. Suppose that we live in a world that can be described by QFT on a curved spacetime of GTR, and suppose further that this world contains a time machine whose operation involves the existence of base points in $\mathrm{H}^{+}(\Sigma)$. Then in principle we would be able to detect the fact that we live in such a world by observing that commutativity fails, $\left[\varphi\left(f_{1}\right), \varphi\left(f_{2}\right)\right] \neq 0$, even when the supports of $f_{1}$ and $f_{2}$ are relatively spacelike when viewed from within the local spacetime regions we inhabit (see Kay et al. 1997).

\section{No-go results from Euclidean quantum gravity}

Cassidy and Hawking (1998) have argued that Euclidean methods provide a "probabilistic" chronology protection result. The ambitions of the Euclidean approach extend

\footnotetext{
53 Krasnikov distinguishes globally hyperbolic subsets from intrinsically globally hyperbolic subsets: a subset $\mathcal{N} \subset \mathcal{M}$ of a spacetime $\mathcal{M}, g_{a b}$ is globally hyperbolic iff (i) $\forall p, q \in \mathcal{N},\left(J^{+}(p) \cap J^{-}(q)\right) \subset \mathcal{N}$ and (ii) strong causality holds in $\mathcal{N}$, whereas $\mathcal{N}$ is intrinsically globally hyperbolic iff $\mathcal{N},\left.g_{a b}\right|_{\mathcal{N}}$ is a globally hyperbolic spacetime. The difference between the two lies in the importance of $\mathcal{M}-\mathcal{N}$ : strong causality fails in $\mathcal{N}$ if a CTC (or almost-CTC) loops through $\mathcal{M}-\mathcal{N}$ and returns to $\mathcal{N}$, but such a set will still be intrinsically globally hyperbolic. The union of globally hyperbolic subsets excludes the chronology violating regions, whereas the union of intrinsically globally hyperbolic subsets can cover a chronology violating spacetime.
} 
beyond those of either QFT on curved spacetimes or the semi-classical approach: techniques borrowed from the path integral formulation of QFT are used to study "full quantum gravity" effects, ranging from the initial singularity to black hole evaporation. ${ }^{54}$ In QFT path integrals are used to calculate the amplitude for a transition from one field configuration to another. These are often mathematically more well-behaved in Euclidean space (obtained by replacing $t$ with $-i t$ in global inertial coordinates) than in Lorentz-signature spacetime, so field theorists frequently use Euclidean path integrals and then analytically continue the relevant functions back to Minkowski space to find S-matrix elements and the like. The Euclidean approach focuses on path integrals like those from QFT, such as that for gravity coupled to a scalar field $\varphi .55$

$$
\mathcal{Z}(\mathcal{M})=\int d \mu\left(g_{a b}\right) d(\varphi) \exp \left(-S_{E}\left[g_{a b}, \varphi\right]\right) .
$$

The analog of the " $t \rightarrow-i t$ " trick in curved space is to replace a Lorentzian metric with a Riemannian metric; the path integral extends over the space of all Riemannian metrics defined over the manifold $\mathcal{M}$. In some cases it is possible to analytically continue the Riemannian metric back into a Lorentzian one to calculate physical quantities.

There are a number of difficulties in interpreting the quantity $\mathcal{Z}(\mathcal{M})$. The tricks for handling the measure over field configurations in perturbative calculations in QFT do not immediately extend to this case, and there is no general definition for the measures $d \mu\left(g_{a b}\right)$ and $d(\varphi)$. The interpretation of the "wave function of the universe" $\Psi\left[h_{i j}\right]$, a complex-valued functional on the space of metrics $h_{i j}$ defined on 3-dimensional manifolds, is contentious; it is not clear whether $\Psi\left[h_{i j}\right]$ can be interpreted as a "probability amplitude" by analogy with QFT. Following the familiar Born rule, suppose we take $\int_{\mathcal{R}}\left|\Psi\left[h_{i j}\right]\right|^{2} d \mu\left(h_{i j}\right)$ to be the probability that the three-geometry of the universe falls within a range of values " $\mathcal{R}$ ". This "naïve interpretation" faces a number of obstacles. ${ }^{56}$ What does it mean to say that the universe has a given three-geometry on a surface $\Sigma$, without specifying a time when this geometry obtains? In addition, there is no guarantee that the dynamical evolution of $\Psi$ has properties that would make it possible to interpret these probabilities as being somehow related to a curve through the space of allowed metrics. The Hartle-Hawking "no boundary" proposal interprets these probabilities as follows: define the ground state wave function(al) of the universe $\Psi\left(h_{i j}, \varphi_{0}, \Sigma\right)$ as a sum of $\mathcal{Z}(\mathcal{M})$ ranging over compact Euclidean manifolds that have a unique boundary $\Sigma$, with induced metric $h_{i j}$ and field configuration $\varphi_{0}$.

\footnotetext{
54 Our comments here about the Euclidean program will be brief; for a careful and thorough assessment of the thorny interpretational issues, see Isham and Butterfield (1999). For a collection of papers introducing the technical apparatus of the Euclidean approach, see Gibbons and Hawking (1993).

55 The Euclidean action for general relativity coupled to a scalar field is given by

$$
S_{E}=-\frac{\pi}{16} \int_{\mathcal{M}} R \sqrt{g} d^{4} x+\frac{\pi}{8} \int_{\partial \mathcal{M}} K \sqrt{h} d^{3} x-\int L(\varphi) \sqrt{g} d^{4} x
$$

where $h:=\operatorname{det}\left(h_{i j}\right), h_{i j}$ being the spatial metric of the boundary of $\mathcal{M}$, written $\partial \mathcal{M} ; K$ is the trace of the extrinsic curvature of $\partial \mathcal{M}$; and $L(\varphi)$ is the Lagrangian of the scalar field.

56 The term is borrowed from Unruh and Wald (1989), who also discuss the difficulties with two other less naïve attempts to introduce probabilities.
} 
The probability calculated using this ground state is interpreted as the probability for the Universe (with an initial spatial geometry $\Sigma, h_{i j}$ ) to "appear from nothing" (Hartle and Hawking 1983).

The Euclidean approach is an initially promising way to study spacetimes with CTCs since some of these spacetimes have well-behaved Euclidean analytic continuations and the approach applies to non-globally hyperbolic spacetimes. Cassidy and Hawking (1998) give a no-go result based on applying the Euclidean approach to a fixed background spacetime. In particular, they study scalar fields with finite temperature on background spacetimes constructed as analytic continuations of periodic Euclidean spaces; i.e., flat Euclidean space with points identified under discrete isometries. These spaces are equivalent to an Einstein universe rotating around some fixed axis; as the rotation rate increases, at a critical value points sufficiently far from the axis rotate faster than $c$. In the original space, the critical value of the boost parameter corresponds to the formation of CTCs. The path integral for the scalar field diverges to infinity at the critical value. At first blush, this result seems to indicate that acausal spacetimes would be incredibly probable according to the no boundary proposal. But an analogy with thermodynamics suggests that a different quantity should be interpreted as the true amplitude: Hawking and Cassidy calculate the entropy of the scalar field, and interpret their result as implying that the density of quantum states tends to zero. This result indicates that the quantum states that would be associated with a chronology violation simply are not available.

Results along these lines could provide a broad basis for chronology protection. They extend more broadly than previous results, since they would outlaw CTCs and not just time machines. But we have two reasons for thinking that the case is not closed. The first relates to the vexed issue of interpreting path integrals and associated probabilities in this setting. We mentioned some of the difficulties associated with probabilities in the Euclidean program above. Cassidy and Hawking's (1998) introduction of thermodynamic considerations muddies the waters further: Can their considerations be consistently combined with assignments of probabilities based on the no-boundary proposal, or do they come into play only in case of divergences? Second, does every acausal spacetime have a Euclidean section? If the answer is no, as we conjecture, the Euclidean techniques do not apply across the board. At best this approach would give a plausibility argument that quantum effects prevent CTCs in spacetimes outside its domain of applicability.

\section{Conclusion}

The physics literature on time machines has evolved in the absence of any precise delineation of the spacetime structure that would characterize the operation of these devices. This seemingly scandalous situation is partly explained by the combination of the facts that most of the physics literature is aimed at producing no-go results and that most of the contributors to the literature have accepted Hawking's dictum that compactly generated Cauchy horizons are a necessary feature of the operation of time machines. Since this dictum is questionable, so are the effectiveness of the no-go results based on it. But, to put a better face on the endeavor, what has become known as 
the time machine literature in physics can be construed as an investigation of the prospects of achieving no-go results showing that, under physically reasonable conditions, CTCs cannot develop in spacetimes initially free of these pathologies. Such chronology protection theorems, if sufficiently general, would also constitute no-go results for time machines, however the vagaries associated with such devices are settled. Our review indicates that this sought after generality has not been achieved. At the same time, the pursuit of chronology protection results has proved to be a fruitful way to probe the foundations of classical GTR and the interface between general relativity and quantum field theory.

Perhaps in the not too distant future it will be possible to discuss the implications of a full quantum theory of gravity for time travel and time machines. But that day is not here. Of the two most promising approaches to the sought after theory-loop quantum gravity (LQG) and string theory - the former is closer to being able to claim the status of a definite theory. ${ }^{57}$ Preliminary results indicate that LQG may be able to eliminate the spacetime singularities that are generic among the solutions to Einstein's field equations. ${ }^{58}$ Perhaps LQG will also eliminate CTCs; but in its present incarnation LQG simply ignores chronology violations since it aims at producing quantum gravity by means of a canonical quantization of classical GTR, and this quantization procedure is only applied to globally hyperbolic spacetimes. Nevertheless, CTCs might emerge in the classical limit of LQG; but at the present time this limit is poorly understood. Note also that LQG eliminates classical general relativistic spacetime singularities not by replacing them with non-singular classical spacetime but by showing that the classical singularity corresponds to a well-defined quantum state that, presumably, does not legitimate the use of a classical spacetime description to any approximation. Eliminating CTCs and time machines as a byproduct of eliminating classical relativistic spacetime structure may strike some (as it strikes us) as less satisfying than a general chronology protection theorem for classical GTR.

The study of time machines is a good opportunity for forging a partnership between physics and the philosophy of physics. Of course, philosophers have to recognize that in this particular instance the partnership is necessarily an unequal one since the mathematical physicists have to do the heavy lifting. But it also seems clear that a little more cooperation with philosophers of science in attending to the analysis of what it takes to be a time machine could have led to some helpful clarifications in the physics literature.

Finally, at the risk of repetition, we want to underscore two points that have not penetrated very far into philosophical consciousness. The first is that the physics literature on time machines engages a set of issues that are largely distinct from those involved in the so-called paradoxes of time travel. More attention to the former by philosophers could reinvigorate discussions of the paradoxes and related issues. The second point is that chronology protection theorems are sufficient but not necessary for showing that a time machine cannot operate; so even if the loopholes in the presently existing chronology protection theorems cannot be plugged, there remain other

\footnotetext{
57 For an overview, see Rovelli (2004).

58 See, for example, Bojowald (2001), but see also Brunnemann and Thiemann (2006).
} 
means for showing that Nature does not sanction the operation of a time machine. Our preferred means would be to show that the Potency Condition cannot be realized in physically interesting models of GTR.

Acknowledgements We are grateful to Robert Geroch, Tim Maudlin, John Norton, Steve Savitt, and Robert Wald for helpful suggestions. Special thanks are due to the participants of a symposium held in honor of Arthur Fine (where an earlier version of this paper debuted) for stimulating discussion and questions. CW's research has been funded in part by the Swiss National Science Foundation project "Properties and Relations"(100011-113688).

\section{References}

Arntzenius, F., \& Maudlin, T. (2005). Time travel and modern physics. In E. N. Zalta (Ed.), Stanford encyclopedia of philosophy. http://plato.stanford.edu/archives/sum2005/entries/time-travel-phys/.

Bojowald, M. (2001). Absence of a singularity in loop quantum cosmology. Physical Review Letters, 86, 5227-5230.

Boulware, D. G. (1992). Quantum field theory in spacetime with closed timelike curves. Physical Review $D, 46,4421-4441$.

Brunnemann, J., \& Thiemann, T. (2006). On (cosmological) singularity avoidance in loop quantum gravity. Classical and Quantum Gravity, 23, 1395-1428.

Carroll, J. W. (1994). Laws of nature. New York: Cambridge University Press.

Carroll, J. W. (2004). Readings on laws of nature. Pittsburgh, PA: University of Pittsburgh Press.

Carroll, S. (2004). Why is the universe accelerating? In W. L. Friedman (Ed.), Measuring and Modeling the Universe. Cambridge: Cambridge University Press. astro-ph/0310342.

Cassidy, M. J., \& Hawking, S. W. (1998). Models for chronology selection. Physical Review D, 57, 2372 2380.

Chrusciel, P. T., \& Isenberg, J. (1993). Compact Cauchy horizons and Cauchy surfaces. In B. L. Hu \& T. A. Jacobson (Eds), Directions in general relativity (vol. 2, pp. 97-107) Cambridge: Cambridge University Press.

Cramer, C. R., \& Kay, B. S. (1996). Stress-energy must be singular on the Misner space horizon even for automorphic field. Classical and Quantum Gravity, 13, L143-L149.

Davies, P. (2002). How to build a time machine. New York: Viking Penguin.

Deutsch, D. (1991). Quantum mechanics near closed timelike lines. Physical Review D, 44, 3197-3271.

Deutsch, D., \& Lockwood, M. (1994). The quantum physics of time travel. Scientific American, 270 (March), $68-74$.

Earman, J. (1995). Bangs, crunches, whimpers, and shrieks: Singularities and acausalities in relativistic spacetimes. New York: Oxford University Press.

Earman, J., \& Wüthrich, C. (2004). Time machines. In E. N. Zalta (Ed.), Stanford Encyclopedia of Philosophy. http://plato.stanford.edu/archives/win2004/entries/time-machine/.

Everett, A. (2004). Time travel paradoxes, path integrals, and the many worlds interpretation of quantum mechanics. Physical Review D, 69:124023.

Fewster, C. J. (2005). Energy inequalities in quantum field theory. math-ph/0501073.

Fewster, C. J., \& Higuchi, A. (1996). Quantum field theory on certain non-globally hyperbolic spacetimes. Classical and Quantum Gravity, 13, 51-61.

Fewster, C. J., \& Wells, C. G. (1995). Unitarity of quantum field theory and closed timelike curves. Physical Review D, 52, 5773-5782.

Ford, L. H., \& Roman, T. A. (1996). Quantum field theory constrains traversable wormhole geometries. Physical Review D, 53, 5496-5507.

Friedman, J. L. (1997). Field theory on spacetimes that are not globally hyperbolic. Fields Institute Communications, 15, 43-57.

Friedman, J. L., Papastamatiou, N. J., \& Simon, J. Z. (1992). Failure of unitarity for interacting fields on spacetimes with closed timelike curves. Physical Review D, 46, 4456-4469.

Friedman, J. L., Schleich, K., \& Witt, D. M. (1993). Topological censorship. Physical Review Letters, 71, $1486-1489$. 
Frolov, V. P. (1991). Vacuum polarization in a locally static multiply connected spacetime and time machine problems. Physical Review D, 43, 3878-3894.

Geroch, R. P. (1970). Domain of dependence. Journal of Mathematical Physics, 11, 437-449.

Geroch, R. P. (1977). Prediction in general relativity. In J. Earman, C. Glymour, \& J. Stachel (Eds.), Foundations of spacetime theories, Minnesota studies in the philosophy of science (vol. 8, pp. 81-93). Minneapolis: University of Minnesota Press.

Gibbons, G. W., \& Hawking, S. W. (1993). Euclidean quantum gravity. Singapore: World Scientific.

Gott, R. (1991). Closed timelike curves produced by pairs of moving cosmic strings: Exact solutions. Physical Review Letters, 66, 1126-1129.

Gott, R. (2001). Time travel in Einstein's universe. New York: Houghton Mifflin.

Hartle, J., \& Hawking, S. W. (1983). Wave function of the universe. Physical Review D, 28, 2960-2975.

Hawking, S. W. (1992a). Chronology protection conjecture. Physical Review D, 46, 603-611.

Hawking, S. W. (1992b). The chronology protection conjecture. In H. Sato, \& T. Nakamura (Eds.), The sixth Marcel Grossmann meeting (pp. 3-13). Singapore: World Scientific.

Hawking, S. W., \& Ellis, G. F. R. (1973). The large scale structure of spacetime. Cambridge: Cambridge University Press.

Hawking, S. W., Thorne, K. S., Novikov, I., Ferris, T., \& Lightman, A. (2002). The future of spacetime. New York: W. W. Norton.

Hiscock, W. A. (2000). Quantized fields and chronology protection. gr-qc/0009061 v2.

Hochberg, D., \& Visser, M. (1997). Geometric structure of the generic traversable wormhole throat. Physical Review D, 56, 4745-4755.

Hochberg, D. \& Visser, M. (1999). General dynamic wormholes and violation of the null energy condition. gr-qc/9901020.

Horwich, P. (1987). Asymmetries in time. Cambridge: MIT Press.

Isenberg, J., \& Moncrief, V. (1985). Symmetries of cosmological Cauchy horizons with exceptional orbits. Journal of Mathematical Physics, 26, 1024-1027.

Isham, C., \& Butterfield, J. (1999). On the emergence of time in quantum gravity. In J. Butterfield (Ed.), The arguments of time (pp. 111-168). Oxford: Oxford University Press.

Kay, B. S. (1992). The principle of locality and quantum field theory on (non-globally hyperbolic) curved spacetimes. Reviews in Mathematical Physics, Special Issue, 4, 167-195.

Kay, B. S. (1997). Quantum fields in curved spacetime: Non global hyperbolicity and locality. In R. Longo et al. (Eds.), Operator algebras and quantum field theory (pp. 578-588). Cambridge, MA: International Press.

Kay, B. S., Radzikowski, M. J., \& Wald, R. M. (1997). Quantum field theory on spacetimes with compactly generated cauchy horizons. Communications in Mathematical Physics, 183, 533-556.

Kim, S.-W. \& Thorne, K. S. (1991). Do vacuum fluctuations prevent the creation of closed timelike curves? Physical Review D, 43, 3929-3957.

Krasnikov, S. V. (1996). Quantum stability of the time machine. Physical Review D, 54, 7322-7327.

Krasnikov, S. V. (1997). Causality violation and paradoxes. Physical Review D, 55, 3427-3430.

Krasnikov, S. V. (1998). A singularity-free WEC-respecting time machine. Classical and Quantum Gravity, 15, 997-1003.

Krasnikov, S. V. (1999). Quantum field theory and time machines. Physical Review D, 59, 024010.

Krasnikov, S. V. (2002). No time machines in classical general relativity. Classical and Quantum Gravity, 19, 4109-4129.

Lewis, D. K. (1973). Counterfactuals. Cambridge, MA: Harvard University Press.

Lobo, F. S. N. (2005). Phantom energy and traversable wormholes. Physical Review D, 71, 084011.

Maeda, K., Ishibashi, A., \& Narita, M. (1998). Chronology protection and non-naked singularities. Classical and Quantum Gravity, 15, 1637-1651.

Manchak, J. (2007). On 'time machines' in general relativity. Unpublished manuscript.

Nahin, P. J. (1999). Time machines (2nd ed.). New York: AIP Press.

Ori, A. (1993). Must time-machine construction violate the weak energy condition? Physical Review Letters, $71,2517-2520$.

Ori, A. (2005). A class of time-machine solutions with a compact vacuum core. Physical Review Letters, 95, 021101.

Ori, A. (2007). Formation of closed timelike curves in a composite vacuum/dust asymptotically flat spacetime. Physical Review D, 76, 044002.

Overbye, D. (2005). Remembrances of things future: The mystery of time. New York Times, June 282005. 
Penrose, R. (1998). The question of cosmic censorship. In R. M. Wald (Ed.), Black holes and relativistic stars (pp. 103-122). Chicago: University of Chicago Press.

Politzer, H. D. (1992). Simple quantum systems with closed timelike curves. Physical Review D, 46, 44704476.

Randles, J. (2005). Breaking the time barrier. New York: Paraview Pocketbooks.

Rovelli, C. (2004). Quantum gravity. Cambridge: Cambridge University Press.

Sushkov, S. S. (1997). Chronology protection and quantized fields: complex automorphic scalar field in Misner space. Classical and Quantum Gravity, 14, 523-533.

Tipler, F. J. (1976). Causality violation in asymptotically flat space-times. Physical Review Letters, 37, 879-882.

Tipler, F. J. (1977). Singularities and causality violation. Annals of Physics, 108, 1-36.

Unruh, W., \& Wald, R. (1989). Time and the interpretation of canonical quantum gravity. Physical Review $D, 40,2598-614$.

Visser, M. (1997). The reliability horizon for semi-classical quantum gravity: metric fluctuations are often more important than back-reaction. Physics Letters B, 415, 8-14.

Visser, M. (2003). The quantum physics of chronology protection. In G. W. Gibbons, E. P. S. Shellard, \& S. J. Rankin (Eds.), The future of theoretical physics and cosmology: celebrating Stephen Hawking's 60th birthday (pp. 161-176). Cambridge: Cambridge University Press.

Vollick, D. N. (1997). How to produce exotic matter using classical fields. Physical Review D, 56, 47204723.

Wald, R. M. (1984). General relativity. Chicago: University of Chicago Press.

Wald, R. M. (1994). Quantum field theory in curved spacetime and black hole thermodynamics. Chicago: University of Chicago Press. 